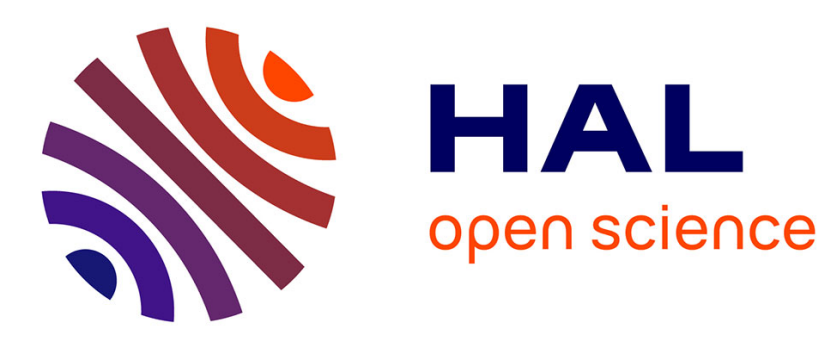

\title{
Defect-mediated turbulence in bubbly Taylor-Couette flow
}

Bruno van Ruymbeke, Noureddine Latrache, Céline Gabillet, Catherine Colin

\section{To cite this version:}

Bruno van Ruymbeke, Noureddine Latrache, Céline Gabillet, Catherine Colin. Defect-mediated turbulence in bubbly Taylor-Couette flow. Physical Review Fluids, 2020, 5 (3), pp.034302. 10.1103/PhysRevFluids.5.034302 . hal-03098317

\section{HAL Id: hal-03098317 https://hal.science/hal-03098317}

Submitted on 5 Jan 2021

HAL is a multi-disciplinary open access archive for the deposit and dissemination of scientific research documents, whether they are published or not. The documents may come from teaching and research institutions in France or abroad, or from public or private research centers.
L'archive ouverte pluridisciplinaire HAL, est destinée au dépôt et à la diffusion de documents scientifiques de niveau recherche, publiés ou non, émanant des établissements d'enseignement et de recherche français ou étrangers, des laboratoires publics ou privés. 


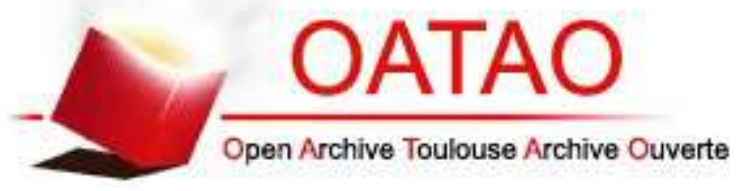

\section{Open Archive Toulouse Archive Ouverte}

OATAO is an open access repository that collects the work of Toulouse researchers and makes it freely available over the web where possible

This is an author's version published in: https://oatao.univ-toulouse.fr/27213

\section{Official URL:}

https://doi.org/10.1103/PhysRevFluids.5.034302

\section{To cite this version:}

Van Ruymbeke, Bruno and Latrache, Noureddine and Gabillet, Céline and Colin, Catherine Defect-mediated turbulence in bubbly Taylor-Couette flow. (2020) Physical

Review Fluids, 5 (3). 034302. ISSN 2469-990X

Any correspondence concerning this service should be sent to the repository administrator: tech-oatao@listes-diff.inp-toulouse.fr 


\title{
Defect-mediated turbulence in bubbly Taylor-Couette flow
}

\author{
Bruno Van Ruymbeke, ${ }^{1}$ Noureddine Latrache $\odot,{ }^{2,},{ }^{*}$ Céline Gabillet, ${ }^{1}$ and Catherine Colin ${ }^{3}$ \\ ${ }^{1}$ IRENav, EA3634-French Naval Academy, Brest, France \\ ${ }^{2}$ Université de Bretagne Occidentale, IRDL/UBO UMR CNRS 6027, Rue de Kergoat, 29238 Brest, France \\ ${ }^{3}$ IMFT, Université de Toulouse, CNRS-INPT-UPS, Toulouse, France
}

(Received 6 May 2019; accepted 3 February 2020; published 9 March 2020)

\begin{abstract}
We investigate experimentally the defect-mediated turbulence (DMT) which is induced by bubbles injection in a Taylor-Couette flow when the inner cylinder is rotating while the outer cylinder is fixed. Bubbles of $1.2 \mathrm{~mm}$ in diameter are injected at the bottom of a Taylor-Couette device of radii ratio equal to 0.91 . The tangential Reynolds number range is $[2200,19300]$ and the air injection rate varies up to $800 \mathrm{ml} / \mathrm{min}$. For these conditions of the experiments, bubbles are trapped in the gap by the Taylor vortices and arranged as patterns (toroidal, wavy toroidal, spirals, and wavy spirals). Visualizations of the bubble patterns were carried out. When decreasing the Reynolds number or increasing the air injection rate, spiral and toroidal patterns can coexist in a composite flow. Defects occur in the bubble's patterns (merging or splitting of the Taylor vortex pairs). By analyzing the space-time diagram of bubbles patterns and their complex demodulation, we highlight different regimes and transitions in the DMT of the bubbly Taylor-Couette flow. The control parameter of the transitions is the air volumetric fraction, which evolves as the ratio between the axial injection Reynolds number and the tangential Reynolds number. By increasing the air volumetric fraction, the defects in the DMT flows are classified as three flow regimes: (i) structured composite flow where the defects are periodic in space and time, (ii) intermittency defects chaos where the defects zones alternate randomly with the patterns in time and space, and (iii) developed defects chaos with a large defects density. The statistical properties of these three regimes of the DMT are analyzed in the framework of the complex Ginzburg-Landau equation.
\end{abstract}

DOI: 10.1103/PhysRevFluids.5.034302

\section{INTRODUCTION}

In the sheared flows, the presence of bubbles in Newtonian liquid is expected to modify the way of the transition to turbulence. Bubbles can modify the turbulence for a wide range of scales from the Kolmogorov scales, up to the large scales of the turbulence. Bubble wakes induced fluctuations can create additional turbulence in turbulent flow [1] or in laminar flow [2]. For some particular conditions, microbubbles, bubbles, or large deformable bubbles can also reduce the shear induced turbulence by interacting with the streak wall turbulence and reduce by this way the wall friction $[3,4]$.

The Taylor-Couette flow that consists of a flow confined in the gap between two coaxial differentially rotating cylinders presents a great interest from a fundamental point of view to study the transition to turbulence. In the transition from laminar to turbulence, the Taylor-Couette flow with the outer cylinder at rest is characterized by streamwise counter-rotating vortices (Taylor vortices), as large as the gap width, separated by inflow and outflow jets, with an axially periodical arrangement. The Taylor vortices are arranged as toroidal patterns. Bubbles can interact with these

*noureddine.latrache@univ-brest.fr 

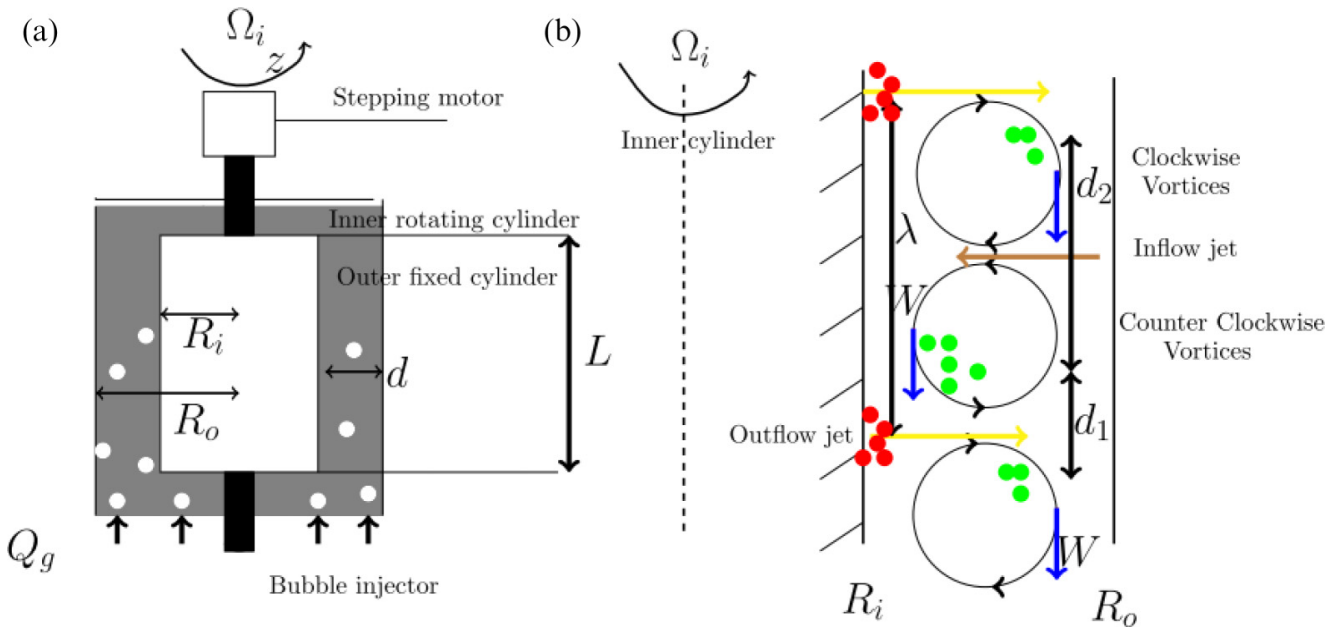

FIG. 1. Conceptual sketch of the bubbly Taylor-Couette flow: (a) Taylor-Couette system; (b) view of preferential localization of the bubbles in the gap (bubbles are represented as full circles).

large-scale vortices. When the outer cylinder is at rest, the Taylor-Couette flow is thus one of the academic sheared flows that is suitable for investigating the effect of bubbles on the transition to turbulence of Newtonian liquid [5,6] and studying the bubbles interaction with the wall friction in a context of bubbly drag reduction [7-9]. For small Reynolds numbers and small Froude numbers, bubbles are accumulated uniformly along the inner cylinder and slide upward along the wall. By increasing the Reynolds number, the Taylor vortices can capture the bubbles. Bubbles are trapped either in the core of the vortices (preferentially in the counterclockwise vortex) or in the outflow jet near the inner rotating cylinder [5,9-12], as represented on the conceptual sketch of Fig. 1. This flow is called the captured bubbles regime.

For the captured bubbles regime, Fokoua et al. [9] have evidenced that a preferential capture of the bubbles in the vortices induces a reduction of the axial wavelength, associated with an increase of the viscous torque at the inner cylinder. On the contrary, a preferential entrapment of the bubbles in the outflow jet leads to an increase of the axial wavelength associated to a reduction in the torque of the inner cylinder. Moreover, it was highlighted that the preferential capture depends on the geometry of the gap (ratio of the outer to the inner cylinders radii $\eta$ ). A small gap plays in favor of bubbles capture by the Taylor vortices, while a large gap enhances bubbles capture by the outflow jet.

Although bubble accumulation modifies the flow by changing the size of the vortices, bubbles can be used as tracers to visualize the arrangement of the Taylor vortices. In Yoshida et al. [13], visualizations of the bubbles carried out simultaneously with the characterization of the Taylor vortices by particle image velocimetry attested that bubbles patterns and Taylor vortices are arranged in the same way. Even though the single-phase flow is characterized by a toroidal arrangement of the Taylor vortices, bubbles can modify this arrangement. At small air injection rate, bubbles patterns still exhibit a toroidal arrangement [9]. But at higher air injection rates, different bubbles patterns such as toroidal or spiral patterns or the combination of these two patterns (i.e., composite flow) have been observed according to the Reynolds number and the air injection rate $[8,13,14]$. Yoshida et al. [13] showed an example of the space-time diagram in the axial direction of the bubbles patterns for the composite flow where the space-time sequences of toroidal and spiral patterns are visible (see Fig. 5(a) in Ref. [13]).

In Van Ruymbeke et al. (2017) [15], based on visualizations of the bubbly pattern, the contribution of cloud bubbles (entrapped in the outflow jet) was discriminated from the contribution of vortices (swirl bubbles). The analysis of preferential positions, azimuthal velocities, and equivalent 
void fraction, of these two kinds of bubbles separately, gave a new insight into the dynamics of the bubble's entrapment for a toroidal arrangement of the Taylor vortices.

In the bubbly Taylor-Couette flow, the spiral pattern comes from bubbles effect under gravity conditions [12]. Note that a spiral arrangement of the Taylor vortices can also be observed in the single Taylor-Couette flow for the outer cylinder at rest when an axial flux is superimposed [16]. In Ref. [8], for low to moderate turbulent Taylor-Couette flows, Murai et al. (2008) [8] examined the link between the viscous torque modification at the inner cylinder and the bubbles patterns. Interestingly enough, it was observed that the torque is minimized for the composite pattern [8].

In the composite flow, the patterns present nucleation of spatiotemporal defects, corresponding to splitting or merging of the Taylor vortices (see Fig. 5(a) in Ref. [13]). Murai et al. (2018) [14] also analyzed the transition between toroidal, and spiral patterns in the composite flow. They inferred that the transition from toroidal to spiral patterns comes from a collapse of the clockwise vortex when bubbles accumulation in the clockwise vortex during the toroidal sequence exceeds a critical value. During the spiral sequence, the gas has been widely evacuated. The transition from spiral to toroidal patterns takes place again when the void fraction is small.

The occurrence of defects in the bubbles patterns was evidenced for the first time by Atken et al. [17] in a highly turbulent Taylor vortex flow, with a superimposed axial flow and ventilation of bubbles at the free surface. Nevertheless, in the study of Atken et al. [17], the composite flow was not identified as a switching regime between toroidal and spiral patterns but as a switching regime between classic traveling Taylor vortices and intermittent pulses of vortices with higher phase velocities. Moreover, in Murai et al. [8,14] and Atken et al. [17], no quantitative information was given about the spatiotemporal defects. To our best knowledge, no detailed investigation of the spatiotemporal defects in a bubbly Taylor-Couette flow has been reported so far.

In the nonlinear theory of the transition to turbulence, the states with spatiotemporal defects are called defect-mediated turbulence (DMT) $[18,19]$. In the framework of this theory, the development of spatiotemporal defects affects the mixing properties of the flow by changing the time and length scales of the flow. The present work is thus focused on the characterization of the spatiotemporal defects occurring in the bubbly Taylor-Couette flow patterns and identification of bubbly parameters that control the development of these defects.

The DMT has been observed in many experiments of driven spatially extended nonlinear systems [20-29]. The DMT is investigated in the theory using the one-dimensional complex GinzburgLandau equation (CGLE) by several authors $[18,30,31]$ :

$$
\frac{\partial A}{\partial t}=A+\left(1+i c_{1}\right) \frac{\partial^{2} A}{\partial z^{2}}-\left(1-i c_{3}\right)|A|^{2} A
$$

where $A$ is the complex order parameter of the field pattern, $t$ and $z$ are, respectively, time and space coordinate while $c_{1}$ and $c_{3}$ represent the linear and nonlinear dispersion coefficients. All plane waves solutions of the CGLE [Eq. (1)] are linearly unstably beyond the Newell line $1-c_{1} c_{3}=0$ to the Benjamin-Feir instability for $c_{3}>1 / c_{1}$ in the plane $\left(c_{1}, c_{3}\right)$ [30]. Beyond the Newell line $\left(c_{3}>1 / c_{1}\right)$, the numerical simulations of the CGLE [Eq. (1)] [30] show the occurence of two chaotic regimes in the plane $\left(c_{1}, c_{3}\right)$ : "phase chaos" without defects and DMT, where the plane waves can present a dislocation of their phase with annihilation of their amplitude $(|A|=0)$. Just beyond their threshold, the nucleation density of defects increases with the driving nonlinear coefficient of dispersion $c_{3}$ of the CGLE [30,31]. In the waves pattern, the presence of defects generates rapid exponential decay of temporal and spatial correlations functions [30]. The inverse of the correlation time is proportional to the density of defects. The mean separation time between two defects is found to be of the same order as the correlation time in the DMT states [30]. Even if the DMT is explored by the CGLE following one-dimensional direction; their properties resemble the ones obtained in the experimental investigations of the flows in three-dimensional directions [25,29].

In the present work, we address the issue of the DMT in the bubbly Taylor-Couette flow for the bubbles captured regime. For the purpose, we have performed visualizations of the bubbles patterns for different Reynolds numbers (based on the rotational velocity) and different air injection rates. 


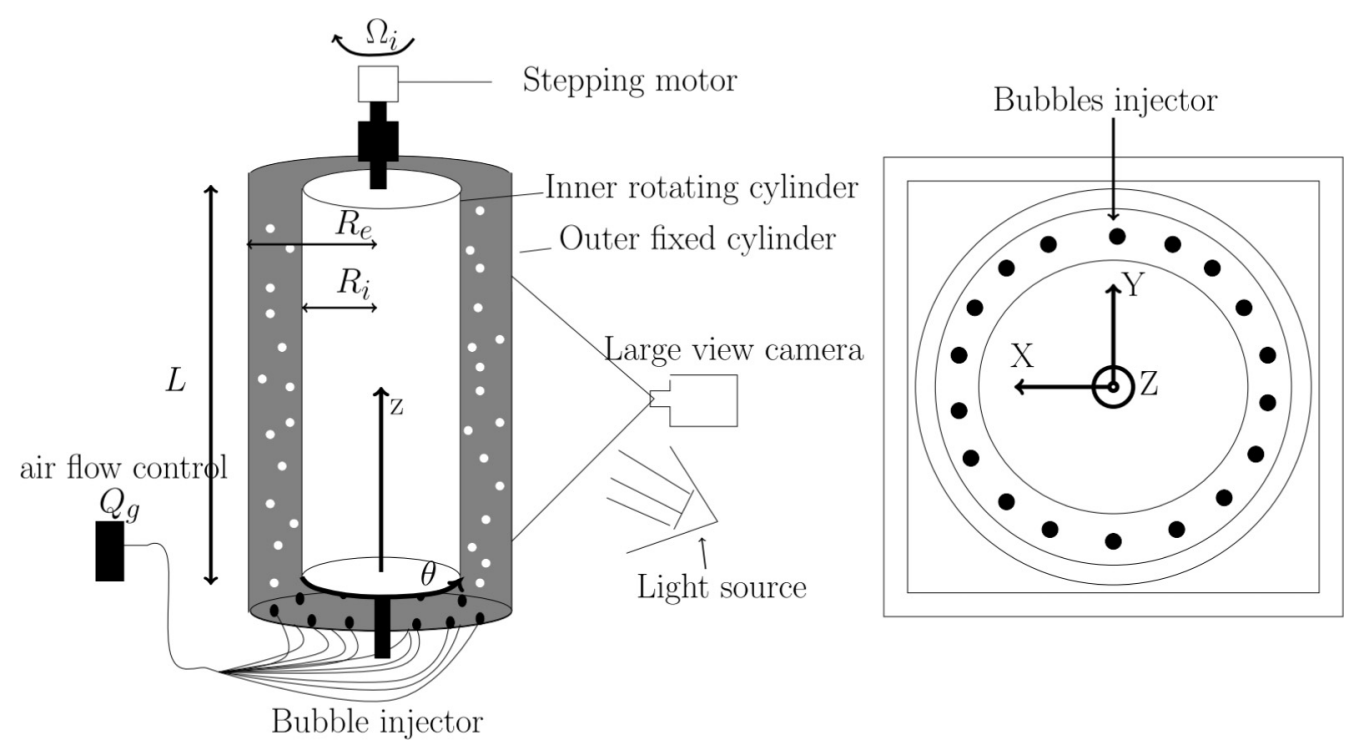

FIG. 2. Scheme of the IRENav Taylor-Couette facility.

The geometry of the device is same as in Fokoua et al. [9] for same bubble size, but the study is performed for higher air injection rates, making possible to observe the different bubbles patterns (not only toroidal pattern but also spiral and composite patterns) for same Reynolds numbers range as in Ref. [9]. The gap's geometry $(\eta=0.91)$ is similar to the one used in Atken et al. [17] but, unlike in Atken et al. [17], the air volume injected in the device is controlled and the study is performed for moderate Reynolds numbers. In our study, we investigate higher Reynolds numbers and higher air injection rates than in Murai et al. (2008) [8]. Unlike in Murai et al. (2008) [8], Yoshida et al. (2009) [13], Van Ruymbeke et al. (2017) [15], Murai et al. (2018) [14] for whom bubbles were preferentially entrapped in the outflow region, in the present study, the geometry of the gap implies preferential capture of the bubbles in the vortices (larger $\eta$ value). This can potentially make a difference in the bubbly patterns development and defects occurrence.

We have carried out statistical analysis of space-time diagrams of the intensity scattered by the bubbles. The bifurcation scenario of the DMT is highlighted by the characterization of correlation time and length and by the characterization of the statistical properties of the defects such as their number, their lifetime, their separation time, and their separation length. The paper is organized as follows: Sec. II describes the experimental setup and flow visualization method. Results are described in Sec. III. Our conclusions are provided in Sec. IV.

\section{EXPERIMENTAL SETUP, CONTROL PARAMETER, AND FLOW VISUALIZATION TECHNIQUE}

The experiments were carried out in the vertical Couette-Taylor device of IRENav composed of concentric inner and outer cylinders (Fig. 2) $[9,11]$. The fixed outer cylinder is made of PPMA with a radius $R_{o}=220 \mathrm{~mm}$. The rotating inner cylinder is made of Plexiglass with a radius $R_{i}=$ $200 \mathrm{~mm}$. The gap's width is $d=20 \mathrm{~mm}$ and the effective height is $L=880 \mathrm{~mm}$. The radii ratio is $\eta=R_{i} / R_{o}=0.9$ and the aspect ratio is $\Gamma_{a}=L / d=44$. Two liquid mixtures of water and glycerol (65\% and $40 \%$ in mass of glycerol) were used in this study.

Spherical air bubbles were injected in the gap at the bottom of Taylor-Couette device through 17 injectors connected to microholes ( $65 \mu \mathrm{m}$ in diameter) equally spaced in the azimuthal direction (Fig. 2). By varying the number of injectors activated and varying the air injection pressure, the input 
gas flow rate $Q_{g}$ was varied between 24 to $800 \mathrm{ml} / \mathrm{min}$ and controlled with an accuracy of $\pm 1.2 \%$. The bubble diameter $d_{b}$ was characterized by high speed video recording and image processing. $d_{b}$ is approximately $1.2 \mathrm{~mm}$ (i.e., $6 \%$ of $d$ ). The terminal rising velocity of the bubbles $V_{b}$ achieved in the motionless mixture can be deduced from a balance between buoyancy and drag forces using the drag coefficient established by Maxworthy et al. [32]. The value of $V_{b}$ in the mixture of $40 \%$ glycerol is twice the value of $V_{b}$ in the mixture of $65 \%$ glycerol.

The varying control parameters of the bubbly Taylor-Couette flow (BTCF) are the angular velocity of the inner cylinder $\Omega_{i}\left(\mathrm{~s}^{-1}\right)$, the kinematic viscosity of the liquid phase $v$, and the air injection rate $Q_{g}$. Based on these control parameters, the BTCF is characterized by the following nondimensional parameters:

The Reynolds number Re compares the inertial centripetal force due to the rotation of the inner cylinder and the viscous force:

$$
\operatorname{Re}=\frac{2 \pi R_{i} \Omega_{i} d}{v}
$$

It is defined as in single-phase flow, because the viscosity is not expected to be modified by the bubbles volume fraction which remains very small.

The Froude number Fr represents the ratio of the inertial centripetal force and the buoyancy force applied on the bubbles:

$$
\mathrm{Fr}=\frac{2 \pi \Omega_{i} R_{i}}{\sqrt{g R_{i}}} .
$$

Fr influences the bubbles capture. The capture occurs for Fr larger than the unity.

The axial Reynolds number of the gas phase $\operatorname{Re}_{g}$ is

$$
\operatorname{Re}_{g}=\frac{Q_{g}}{2 \pi R_{i} v} .
$$

The volumetric fraction $\alpha$ of the gas injected in the gap which is defined as the ratio of the volume of air injected during one rotating period to the volume of the liquid contained in the device:

$$
\alpha=\frac{Q_{g}}{2 \pi R_{i} \Omega_{i} d L}=\frac{\operatorname{Re}_{g}}{\operatorname{Re}} \frac{2 \pi R_{i}}{L} .
$$

$\alpha$ controls the quantity of air available in the gap, according to the rotation velocity. $\alpha$ evolves as the ratio between the Reynolds numbers $\mathrm{Re}$ and $\mathrm{Re}_{g}$.

We can also define the bubbles Reynolds number based on the bubbles terminal rising velocity and their diameter:

$$
\operatorname{Re}_{b}=\frac{V_{b} d_{b}}{v}
$$

The ratio $\operatorname{Re}_{b} / \operatorname{Re}$ (i.e., the ratio between the bubble terminal rising velocity $V_{b}$ and the inner cylinder's tangential velocity $V_{i}$ ) is expected to control the bubbles entrapment inside the gap (preferentially in the Taylor vortices or in the outflow region near the inner cylinder). But the best nondimensional parameter that controls the axial entrapment of the bubbles is the parameter $C$ $[9,10,12]$, defined in Eq. (7). $C$ is the ratio between the axial velocity of the Taylor vortices $W$ and the bubble terminal rising velocity $V_{b}$. $C$ depends on $\operatorname{Re}_{b} / \mathrm{Re}, \mathrm{Re}$, and $\eta$. The higher the $C$ value, the most important the bubbles entrapment in the gap:

$$
C=\frac{W}{V_{b}} .
$$

$H_{\text {new }}$ is the ratio between the centripetal force induced by the Taylor vortices and the centripetal force due to the inner cylinder rotational velocity. By taking into account the axial wavelength $\lambda$ in 


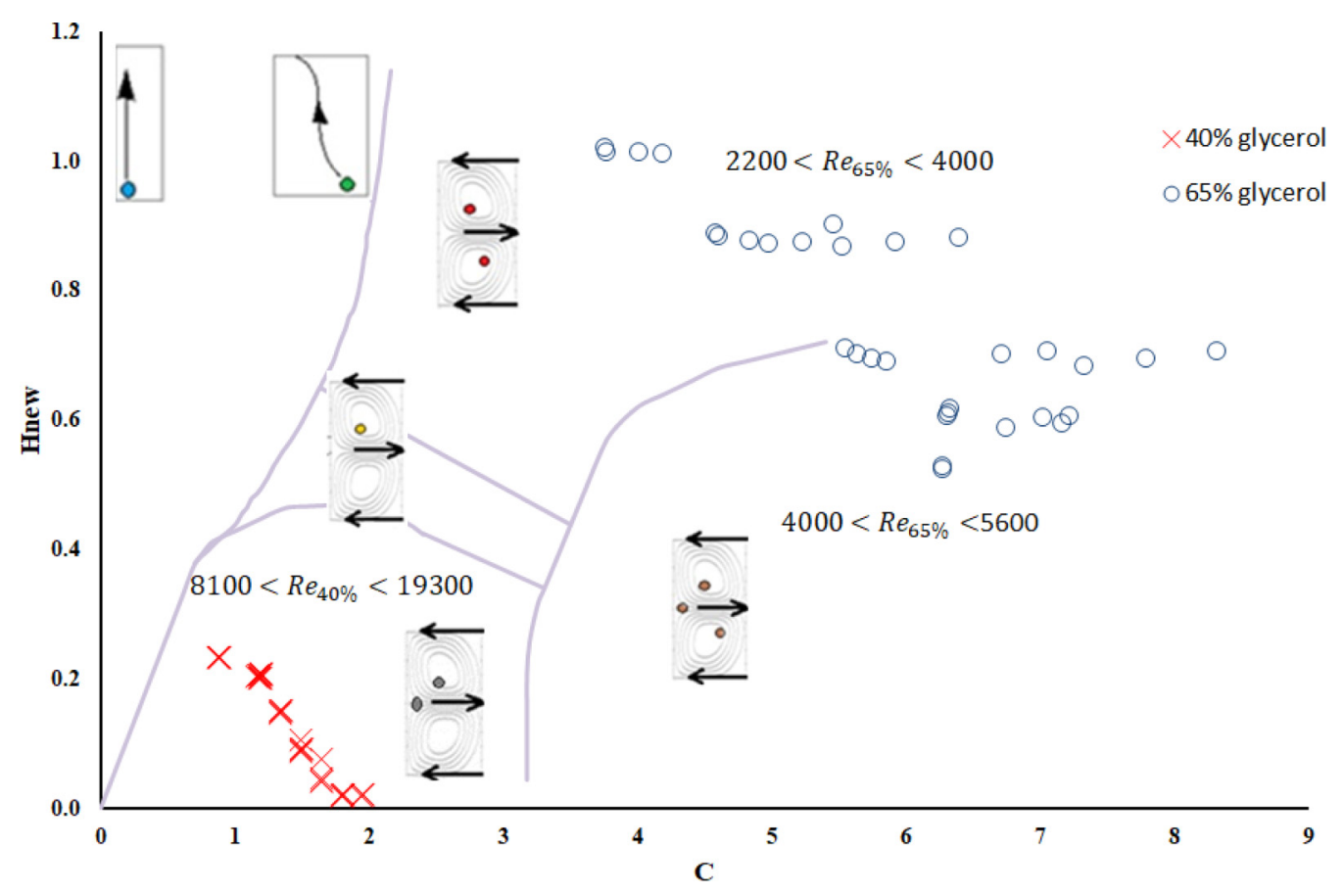

FIG. 3. Map of the bubbles localization in the gap as a function of parameters $C$ and $H_{\text {new }}$ Fokoua et al. [9].

this formulation, Fokoua et al. [9] established a universal map of the bubbles localization in the gap, with regard to parameters $C$ and $H_{\text {new }}$ (Fig. 3):

$$
H_{\text {new }}=4 \frac{W^{2}}{V_{i}^{2}} \frac{R_{i}^{2}}{\lambda^{2}} .
$$

The parameters range of the experimentation are reported in Table I. For the geometry of our Taylor-Couette device, the Taylor vortices occur above a critical value of the Reynolds number $\operatorname{Re}_{c 1}=137$ [33]. The use of the two mixtures makes it possible to cover a large range of the Reynolds number Re. The Re range of the study here is [2 200-5600] and [8 160-19300] for the mixtures of $65 \%$ and $40 \%$ glycerol, respectively, which corresponds in the single-phase flow to the transition from the early turbulent regime to the turbulent regime with persistence of the Taylor vortices [33].

For this range of Reynolds numbers, in the single-phase flow, the Taylor vortices are arranged as steady toroidal patterns, periodically spaced in the axial direction, and no azimuthal wave was observed [33]. No defects are present in the single-phase flow. The axial wavelength measured by Fokoua et al. [9] in the single-phase flow is plotted according to Re number in Fig. 12.

The air volumetric fraction $\alpha$ varies up to $0.07 \%$. Figure 3 shows the operating points of the present study superimposed on the map of Fokoua et al. [9]. For the range of $H_{\text {new }}$ and $C$ values of the present study, bubbles are trapped by the vortices. For the mixture of $65 \%$ glycerol and $\operatorname{Re}<4000$, the bubbles are trapped in both vortices; and for $\operatorname{Re}>4000$, they are captured in both vortices and in the outflow region. For the mixture of $40 \%$ glycerol, bubbles are trapped in the counterclockwise vortex and in the outflow region, they are not captured by the clockwise vortex.

As a consequence of the bubbles capture, the bubbles were used as tracers to visualize the organization of the Taylor vortices in the bubbly two-phase flow. The reflected intensity of the bubbles flow patterns was recorded at $500 \mathrm{f} / \mathrm{s}$ in a front view plane $(\theta, z)$ with a Photron Fastcam 
TABLE I. Geometric specifications of the Taylor-Couette device, physical properties of the test fluid, and setting parameters of the bubbly Taylor-Couette Flow (BTCF).

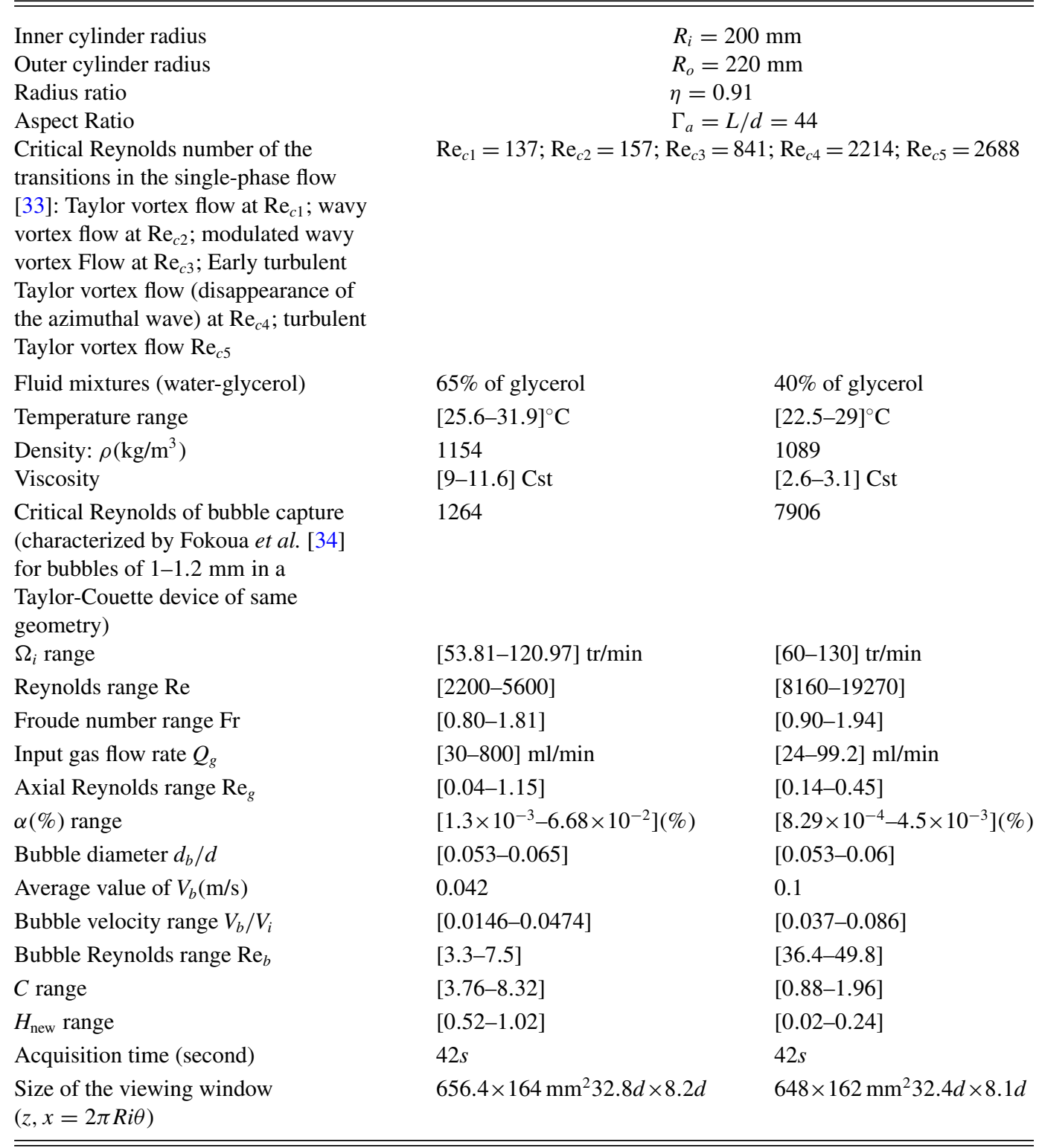

Sa3 120K high-speed camera with 256 grayscales (Fig. 2). The size in the axial direction ( $z$ ) of the visualization window was, respectively, $32.8 d$ for the $65 \%$ mixture and $32.4 d$ for the $40 \%$ mixture, corresponding to 1024 pixels. By the help of a spot light, the contrast was improved between the light reflected by bubble and the black inner cylinder and gives the best visualization of the bubbles flow patterns [Fig. 4(a)].

The bubbly patterns were analyzed following the spatiotemporal technique. For this purpose, at each time step, from each image $I(z, x)$, we have extracted at midlength in the azimuthal direction of the viewing window one intensity line $I(z, x=$ mid-window) [Fig. 4(a)]. The chronological superposition of these lines $I(z, x=$ mid-window $)$ obtained at regular time interval $(1 / 500 \mathrm{~s})$ 


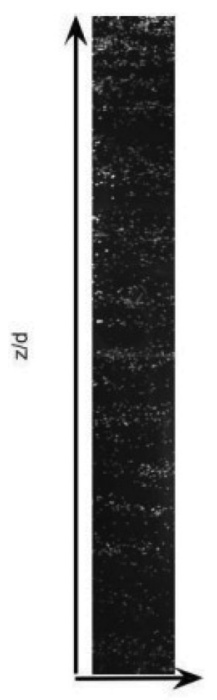

(a) $x$
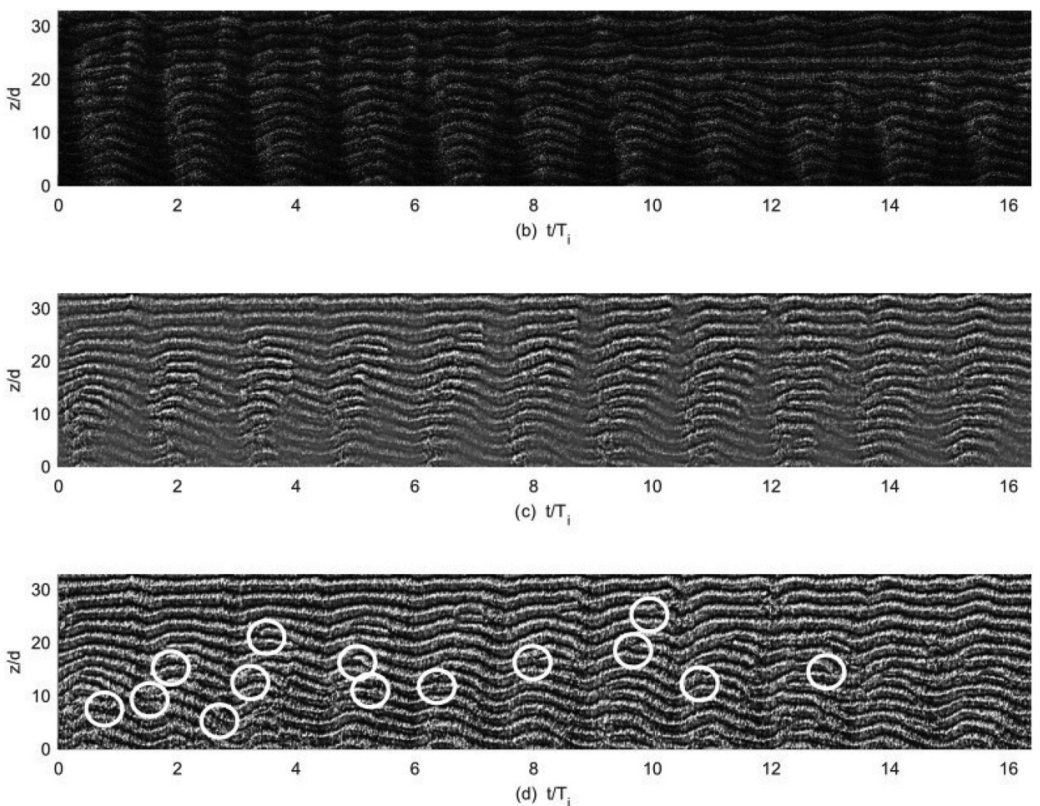

FIG. 4. Visualization of the bubble patterns in the $65 \%$ glycerol mixture for $\operatorname{Re}=2648 ; \operatorname{Re}_{g}=0.16 ; Q_{g}=$ $160 \mathrm{ml} / \mathrm{min}$; $\alpha=0.0088 \%$ : (a) instantaneous image, (b) space-time diagram, (c) first filtered diagram, and (d) homogenized diagram with defects (circle).

provides a space-time diagram $I(z, t)$ [Fig. 4(b)]. In the space-time diagrams, we observe regions with high intensity (bright color) that correspond to captured bubbles by the Taylor vortices and regions with low intensity (dark color) that correspond to liquid phase.

Figure 4(b) presents one example of a space-time diagram of the composite flow made of the wavy toroidal and wavy spiral patterns. Time $t$ is systematically scaled by $T_{i}$, the period of rotation of the inner cylinder $\left(T_{i}=1 / \Omega_{i}\right)$, while the axial distance is scaled by the gap's width $d$. In the space-time diagram of the Fig. 4(b), the axial distance between two bright patterns is representative of the axial distance between a pair of counter-rotating vortices.

With the occurrence of a propagative azimuthal wave, there is a preferential accumulation of the bubbles at the crest of the wave, leading to a nonhomogenous distribution of bubbles in the azimuthal direction $[10,15]$. This is visible by the alternance with time of regions of higher intensity at the crest and lower intensity at the trough (holes) in a same bright pattern. To better highlight the bubbles patterns characteristics (axial wavelength, frequency, phase velocity, ...), a special image post-processing is used according the following two steps:

(1) A two-dimensional Gaussian filter in Fourier coordinates $[\widehat{I}(k, f)]$ was applied to the spacetime diagram $I(z, t)$. This filter has the following characteristics: $f \in\left[0.01 f_{p}, 10 f_{p}\right]$ and in $k \in$ $\left[0.1 k_{p}, 4 k_{p}\right]$, where $k_{p}$ and $f_{p}$ are, respectively, the principal wave number and frequency of the pattern. It enables to merge bubbles strings trapped on both sides of the outflow jet [see Fig. 1(b)] and keep the large-scale structure of the patterns [Fig. 4(c)].

(2) To reduce the difference of the light intensity between the crest (high intensity) and the trough (low intensity) of the azimuthal wave, a homogenization procedure of the intensity is applied to the filtered space-time diagram of Fig. 4(c). The space-time diagram is sampled on $n$ small windows with a size of $2 d$ in space and of $0.064 \mathrm{~s}$ in time. In each small window, the light intensity is rescaled in gray level from 0 to 255 . This procedure permits to converge the light intensity of the holes (trough) to the high intensity of the crest.

In Fig. 4(d), we observe the composite pattern obtained after filtering and homogenization procedures. After applying the homogenization procedure, the intensity close to zero corresponds 
clearly to the inflow regions where the bubbles are not accumulated, while the intensity larger than zero corresponds to outflow regions. For some particular flow conditions, the homogenized spacetime diagram highlights the occurrence of spatiotemporal defects characterized by the disappearance of a pair of vortices (i.e., outflow merging) or the birth of a new pair of vortices (outflow splitting). With this procedure of the homogenization, although the intensity of the troughs of the azimuthal wave is not perfectly equal to the intensity of the crests, defects are clearly distinguished from holes. In the next section, we will discuss about the results.

\section{RESULTS}

In this section, we will present the different patterns obtained according to the control parameters $\left(\mathrm{Re}, \mathrm{Re}_{g}\right)$. The evolution of the frequency and wavelength of the different patterns will be presented with the control parameter $\alpha$. We will pay a particular attention to the analysis of the DMT properties with regard to the control parameter $\alpha$ : evolution of the number of defects, correlation time and length, separation time between two defects, lifetime of defects. The transitions of the different regimes in the DMT will be presented in this section.

\section{A. Flow regimes}

In the bubbly flow, the basic patterns we observe are: toroidal [denoted as $\mathrm{T}$, example is given in Fig. 5(a)], wavy toroidal [denoted as WT, example is given in Fig. 5(b)], spiral [denoted as S, example is given in Fig. 5(c)], or wavy spiral patterns [denoted as WS, example is given in Fig. 5(d)]. The wavy patterns are characterized by strong axial oscillations of the bubbles patterns in time and in the azimutal direction. As can be seen in Figs. 5(b) and 5(d), the wavy patterns are characterized by a dissymmetry in the azimuthal wave, the duration from the crest to the trough being larger than the duration from the trough to the crest. This is due to the upward motion of the bubbles under gravity effect. The toroidal (wavy and nonwavy) patterns correspond to axially periodically spaced horizontal rings of air bubbles trapped in the Taylor vortices. The spiral (wavy and nonwavy) patterns are characterized by strongly inclined stripe bubbles patterns. For the spiral patterns, the Taylor vortices are connected through a single upward bubbles helicoidal path. For the spiral pattern, the slope of the inclined stripes is representative of the axial traveling velocity of the bubbles (axial phase velocity) $V_{\text {phase }}$, which is limited it upper value at $V_{b}$. The periodic axial distance between the stripes is representative of the axial pitch of the helicoid, while the time between two stripes is representative of the time period of the helicoid. As can be seen in Figs. 5(c) and 5(d), the spiral and wavy spiral patterns are characterized by a time period of the helicoid of $2 T_{i}$, which means that the azimuthal traveling velocity of the bubbles in the helicoidal path is $\beta V_{i}$ with $\beta \approx 0.5$ in agreement with bubbles velocity in the bulk. The establishment of the spiral pattern depends on gravity effect. It requires that the axial wavelength equals the axial pitch of the bubbles helicoidal path [8]. For our geometry of the Taylor-Couette device, by assuming that bubbles are moving azimuthally in the gap at an azimuthal velocity of $\beta V_{i}(0<\beta<1)$, and that the bubbles are moving axially at $V_{\text {phase }}$, the establishment of the spiral pattern requires the following condition of the axial wavelength $\lambda$ :

$$
\frac{\lambda}{d}=\frac{\text { axial pitch }}{d} \approx \frac{-2 \pi \eta}{\beta(1-\eta)} \frac{V_{\text {phase }}}{V_{i}} .
$$

Figures 6(a)-6(d) show space-time diagrams of bubbles that depict a composite flow which derives from a combination in space and time of basic patterns: wavy or nonwavy, toroidal, and spiral patterns.

At the junction between toroidal and spiral patterns, defects occur. The composite patterns flow, for which spatial and temporal punctual defects are observed periodically in time and space [Figs. 6(a) and 6(b)] is considered as a structured composite pattern flow (SCP). Unstructured composite patterns flows [Figs. 6(c) and 6(d)] can be discriminated in different flow regimes that we will describe hereafter. 


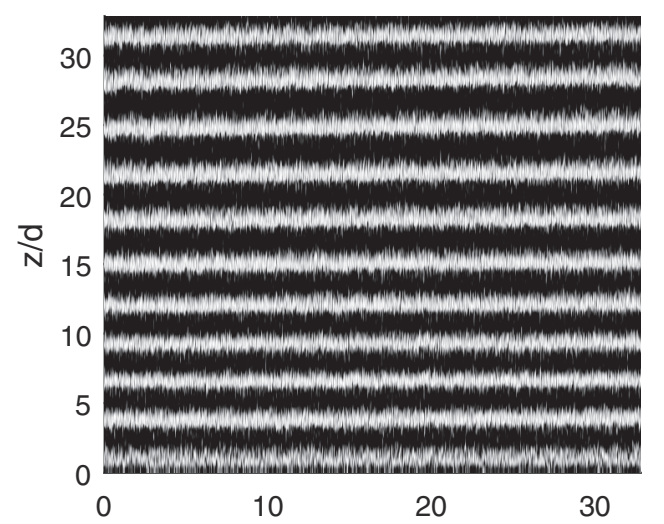

(a) $t / T_{i}$

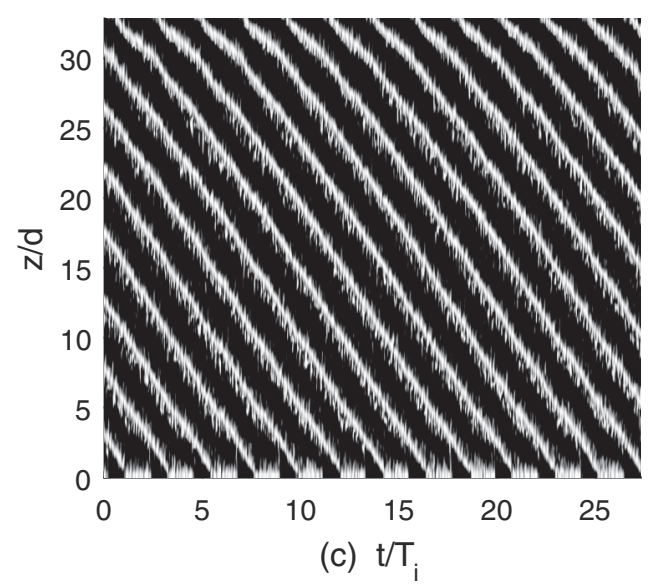

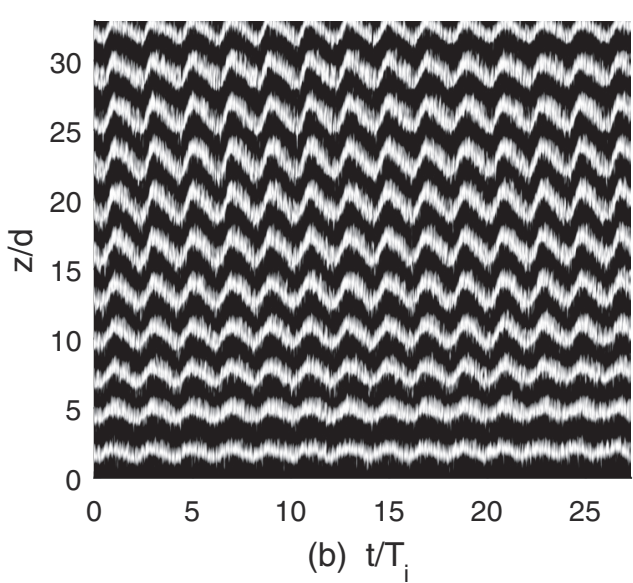

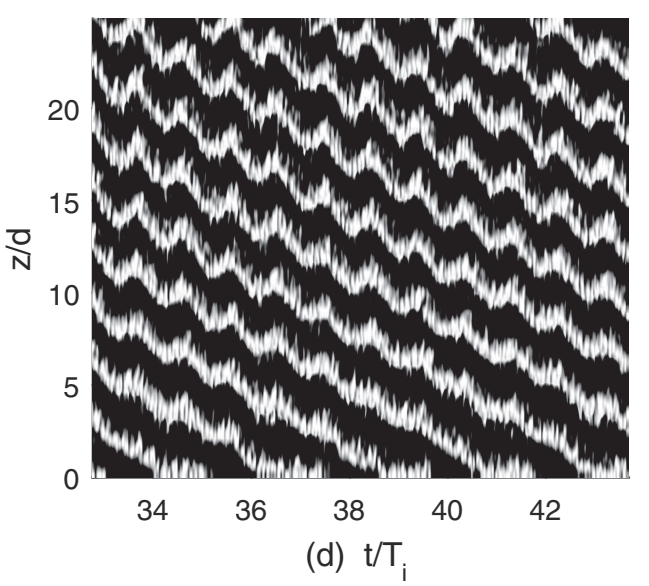

FIG. 5. Examples of space-time diagrams sequences that evidence different bubbly basic patterns. Examples are shown for the mixture of $40 \%$ glycerol: (a) toroidal pattern $\mathrm{T}\left(\operatorname{Re}=8156 ; Q_{g}=32 \mathrm{ml} / \mathrm{min}\right.$; $\left.\operatorname{Re}_{g}=0.13 ; \alpha(\%)=0.0024\right)$; (b) wavy Toroidal pattern WT $\left(\operatorname{Re}=17000 ; Q_{g}=40 \mathrm{ml} / \mathrm{min} ; \operatorname{Re}_{g}=0.19\right.$; $\alpha(\%)=0.0016)$; (c) spiral pattern S $\left(\operatorname{Re}=14500 ; Q_{g}=60.8 \mathrm{ml} / \mathrm{min} ; \operatorname{Re}_{g}=0.28 ; \alpha(\%)=0.0027\right)$. The axial phase velocity determined by the slope of the stripes is $V_{\text {phase }}=0.071 \mathrm{~m} / \mathrm{s} ; V_{\text {phase }} / V_{b}=0.72$; (d) wavy spiral pattern WS $\left(\operatorname{Re}=11361 ; Q_{g}=80 \mathrm{ml} / \mathrm{min} ; \operatorname{Re}_{g}=0.36 ; \alpha(\%)=0.0045\right)$. The axial phase velocity is $V_{\text {phase }}=0.039 \mathrm{~m} / \mathrm{s} ; V_{\text {phase }} / V_{b}=0.41$.

In Fig. 7, we have plotted the different bubbly patterns regimes observed in the $\left(\operatorname{Re}, \operatorname{Re}_{g}\right)$ plane for both mixtures. Some characteristic isovalues of the air volumetric fraction $\alpha$ are superimposed. Generally speaking, in the bubbly flow, we observe that both an increase in the Reynolds number Re or a increase in the air injection rate $\left(\operatorname{Re}_{g}\right)$ play in favor of the occurrence of the azimuthal wave, by increasing the effective gas volume fraction. In Van Ruymbeke et al. (2017) [15], it was claimed that the azimuthal wave was driven by swirl bubbles localized inside the vortices. From our diagram of Fig. 7, it is now clear that this is the increase in the effective gas volume fraction of bubbles entrapped in the counterclockwise vortex which is responsible for the occurrence of this wave. The azimuthal wave is a mean for the flow to evacuate the excess of bubbles trapped in the Taylor vortices at its crest. As shown by Van Ruymbeke et al. (2017) [15], the gas volume fraction is maximum and the azimuthal velocity of the bubbles is minimum at the crest of the azimuthal wave. Bubbles jump axially from a Taylor vortex pair to the next upper pair at the crest of the azimuthal wave. A 


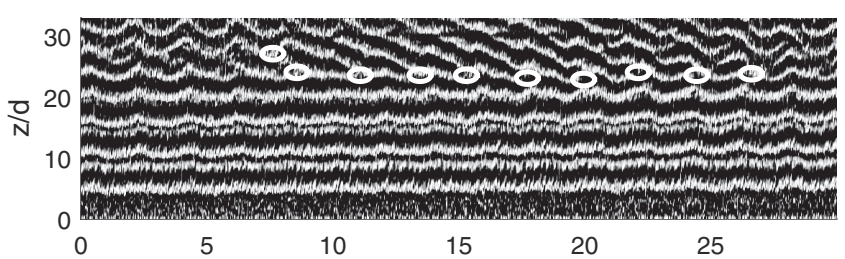

(a) $t / T_{i}$

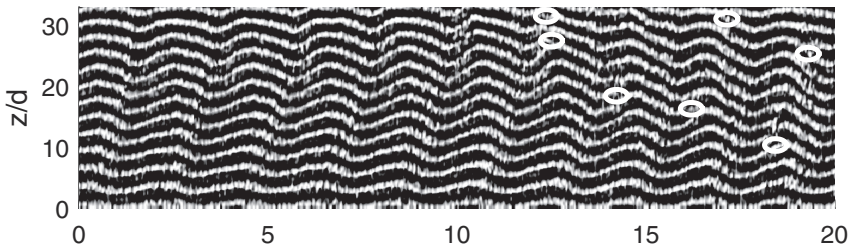

(b) $t / T_{i}$

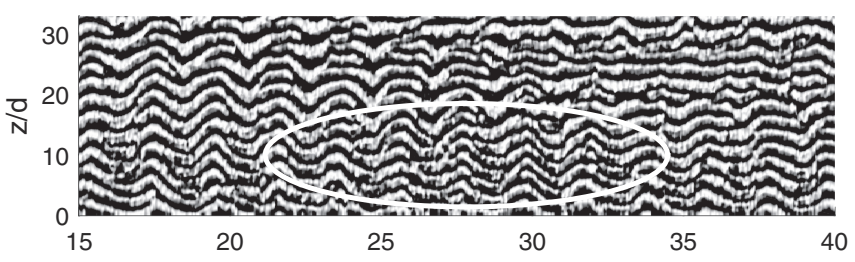

(c) $t / T_{i}$

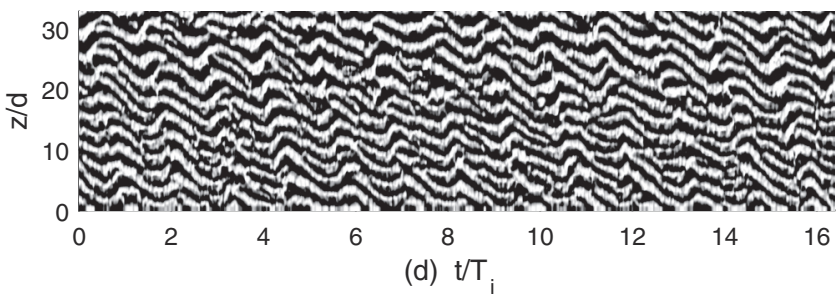

FIG. 6. Examples of space-time diagrams that evidence the different bubbly composite patterns regimes: (a) Structured composite patterns regime $\operatorname{Re}=8339 ; Q_{g}=56 \mathrm{ml} / \mathrm{min} ; \alpha(\%)=0.0042 ; \operatorname{Re}_{g}=0.24 ; 40 \%$ of glycerol, highlights periodical punctual defects in circles. (b) Structured composite patterns regime $\operatorname{Re}=2478$; $Q_{g}=51 \mathrm{ml} / \mathrm{min} ; \alpha(\%)=0.0031 ; \mathrm{Re}_{g}=0.054 ; 65 \%$ of glycerol, highlights periodical punctual defects in circles. (c) Intermittency defect chaos regime, $\operatorname{Re}=3714 ; Q_{g}=160 \mathrm{ml} / \mathrm{min} ; \alpha(\%)=0.0065 ; \operatorname{Re}_{g}=0.17$; $65 \%$ of glycerol, highlights burst of defect in ellipse. (d) Developed defect chaos regime, $\operatorname{Re}=3669 ; Q_{g}=$ $800 \mathrm{ml} / \mathrm{min} ; \alpha(\%)=0.038 ; \mathrm{Re}_{g}=0.98 ; 65 \%$ of glycerol, no basic patterns identified beyond one rotation period.

jump of a moderate number of isolated bubbles will not connect the Taylor vortex pairs and will not introduce defects. An example of isolated bubbles jumping is displayed in Fig. 8. Moderate bubbles jumping is visible on space-time diagrams of the bubbles brightness before applying filtering and homogenization procedures.

For the mixture of $40 \%$ glycerol, $\operatorname{Re}>12000$ and $\alpha<0.0022 \%$, the BTCF is rather a unique pattern flow: the preferential bubble pattern is the wavy toroidal pattern. For this mixture, for $\operatorname{Re}<12000$ or $\alpha>0.0022 \%$, the flow is preferentially a SCP flow.

For the mixture of $40 \%$ glycerol, the axial pitch induced by the bubbles rising path is adjusted to the axial wavelength at one Reynolds number $(\operatorname{Re}=14500)$. At $\operatorname{Re} \approx 14500$, considering the axial phase velocity achieved $\left(V_{\text {phase }} \approx 0.071 \mathrm{~m} / \mathrm{s}\right)$, Eq. (9) is satisfied only for $\beta \approx 0.5$. The occurrence of the spiral also requires that the effective gas volume fraction of the bubbles trapped in the Taylor vortices exceed a critical value, as described by Murai et al. (2018) [14]. For our geometry, we 


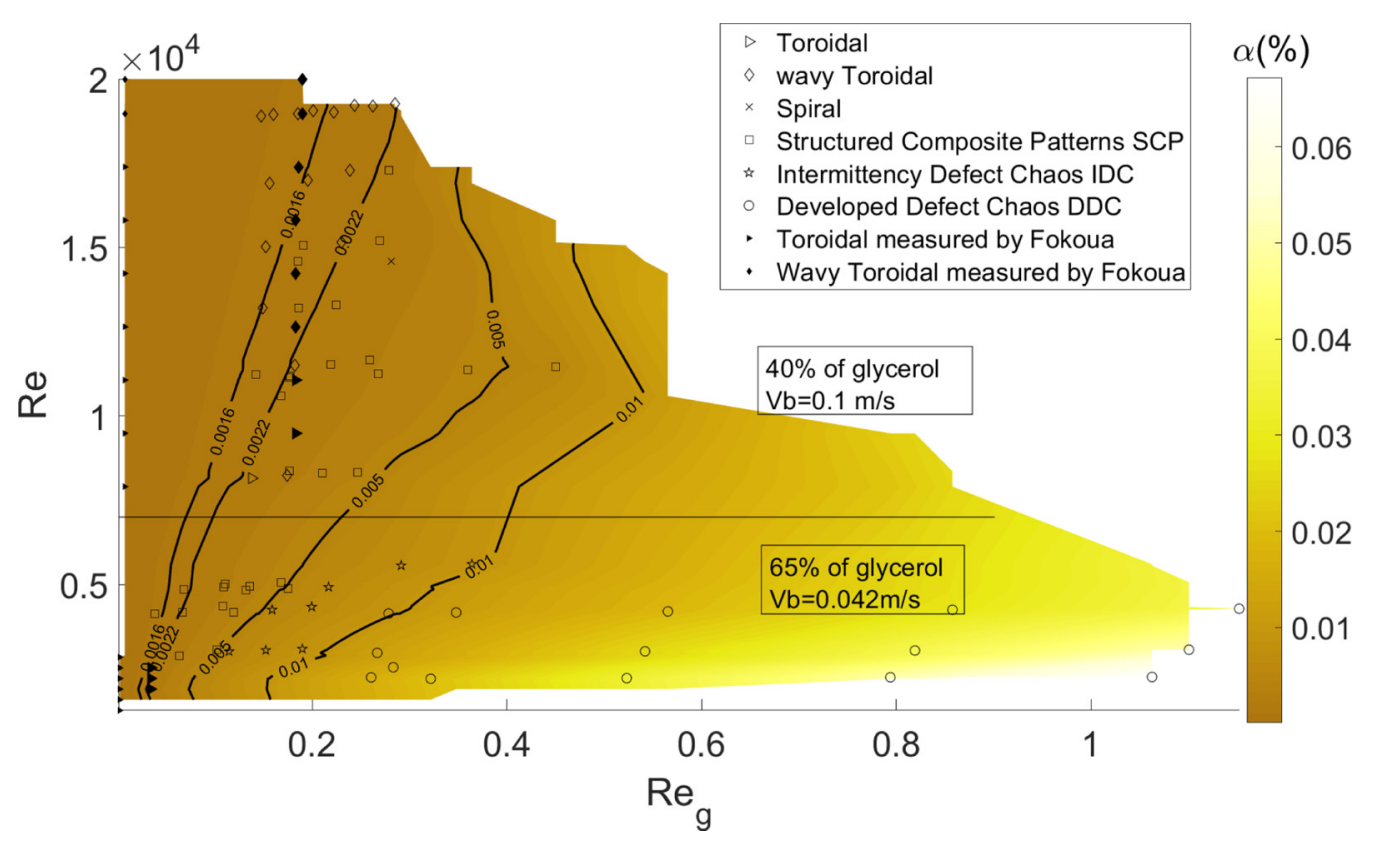

FIG. 7. Map of the bubbly patterns regimes in the $\left(\operatorname{Re}, \mathrm{Re}_{g}\right)$ plane. Isocontours of characteristic values of the parameter $\alpha$ are also plotted with lines and colors.

conclude that the establishment of the steady spiral requires a minimum volumetric fraction $\alpha$ of $0.0027 \%$ at $\operatorname{Re}=14500$. For smaller volumetric fraction, at this Reynolds number, the effective gas volume fraction in the vortices is too small to ensure the steady spiral to develop and we observe a switching between the toroidal and spiral space-time sequences (SCP). For lower or higher Reynolds numbers, the axial wavelength of the Taylor Vortices is too small or too large, respectively, to enable the steady spiral and a SCP is rather observed when $\alpha>0.0027 \%$. Generally speaking, the composite patterns flow takes place when the spiral is the best solution for the excess of bubbles to be expelled while the axial wavelength of the Taylor vortices is too small, not in agreement with the

(a)

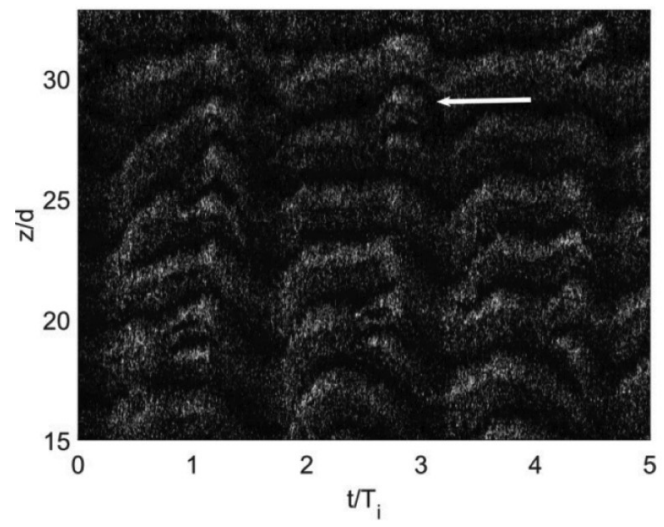

(b)

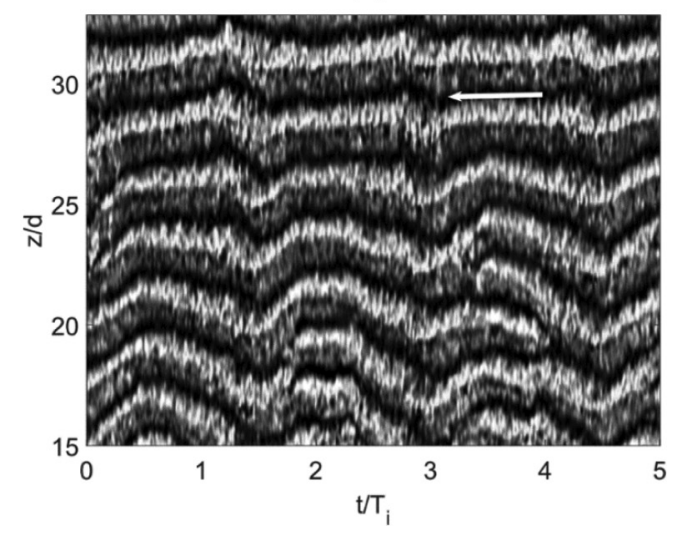

FIG. 8. Bubbles jumping on a zoom of the space-time diagrams of the bubbles brightness: (a) before applying filtering and (b) after applying filtering and homogenization procedures. 


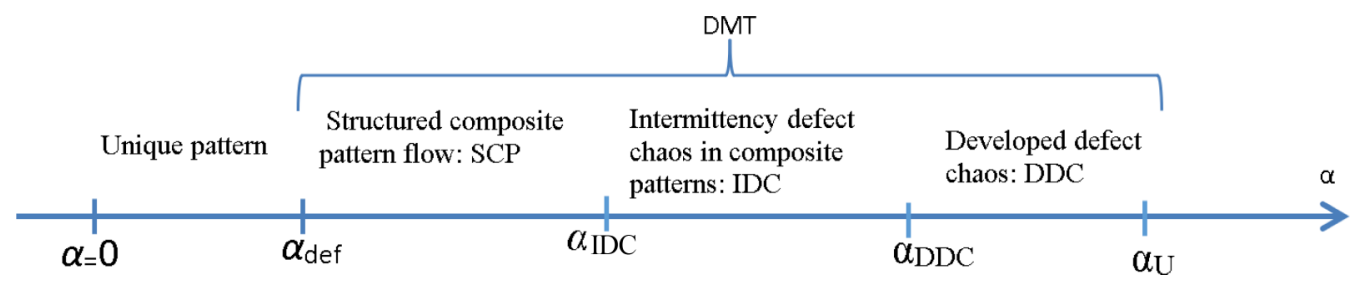

FIG. 9. Sketch that summarizes the transitions from the unique pattern regime to the developed defect chaos regime in the BTCF (for $2200<\operatorname{Re}<5600$ in the explored region of the map of Fig. 7 for the $65 \%$ glycerol mixture). $\alpha_{\text {def }}$ is the critical value of $\alpha$ beyond which the first defect occurs. This value will be characterized in the part dedicated to the statistical analysis of the defects. $\alpha_{U}=0.07 \%$ is the lower value of $\alpha$ of the unexplored yet zone.

axial pitch of the bubble helicoidal path, to enable a connection between the Taylor vortices. For the mixture of $65 \%$ glycerol, although the gravity effect is less important than for the mixture of $40 \%$ glycerol $\left(V_{b} / V_{i}\right.$ smaller), the axial wavelength of the Taylor vortices remains too small compared with the helicoidal pitch.

For the mixture of $65 \%$ glycerol, and for the $\mathrm{Re}$ and $\mathrm{Re}_{g}$ ranges of the present study, the flow is a composite patterns flow, composed of wavy basic patterns. For the mixture of $65 \%$ glycerol, despite the fact that the gravity effect $\left(V_{b} / V_{i}\right)$ is comparable to Murai et al. (2008) [8], the difference in the geometry ( $\eta$ larger than in Murai et al. $[8,14]$ ) makes the spiral pattern unstable. Indeed, in the geometry of Murai et al. $[8,14]$, the entrapment of the bubbles preferentially near the inner cylinder and the increase of the axial wavelength in the bubbly flow make it easier the establishment of a steady spiral; this is not the case for the geometry of the present study which is characterized by preferential entrapment of the bubbles at midgap by the vortices and decrease of the axial wavelength induced by the bubbles. For the mixture of $65 \%$ glycerol,when increasing $\operatorname{Re}_{g}$ (i.e., when increasing the air volumetric fraction $\alpha$, the composite flow is less and less structured with more and more defects occurrences and transitions from different flow regimes are identified: For $\alpha<\alpha_{\text {IDC }}=0.005 \%$, the flow is a SCP flow [Fig. 6(b)]. For $\alpha>\alpha_{\text {IDC }}=0.005 \%$, the SCP flow with punctual defects transits to the regime of the intermittency defect chaos (IDC). For the IDC regime, the defects appear randomly in space and time. They are no more localized in space and time, thus leading to defects spots that alternate in time and space with basic patterns [Fig. 6(c)]. Defects spots are identified with space and time sequences where neither the toroidal, nor the spiral patterns prevail. A further increase in $\alpha$ above the critical value of $\alpha_{\mathrm{DDC}}=0.01 \%$, leads to the developed defect chaos regime: DDC [Fig. 6(d)] for which the number of defects is high and for which the basic patterns do not persist beyond a time period of the azimuthal wave. In this case, there is a continuous switching between toroidal and spiral patterns. Interesting enough is the fact that Murai et al. (2008) [8] in the range of $\left(\mathrm{Re}, Q_{g}\right)$ of their study did not observe unstructured composite flows (IDC or DDC). As mentioned before, the main difference between the two studies lies in the difference in the gap's geometry. Indeed, the geometry of the present study does not enable the development of a stable spiral pattern, which promotes the continuing switching between spiral and toroidal patterns (transition to DDC regime). The increase in the effective volume fraction (by the increase of $Q_{g}$ ) destabilizes the toroidal pattern in favor of the spiral pattern; the increase in the axial pitch of the helicoidal bubble path (by the decrease of $V_{i}$ ) destabilizes the spiral pattern in favor of the toroidal pattern. As a consequence, an increase in the air volumetric fraction $\alpha$, which is the ratio between $\operatorname{Re}_{g}$ and $\operatorname{Re}$ is expected to destabilize both the spiral and toroidal patterns, thus leading to more and more defects occurrence.

The air volumetric fractions $\alpha$ seems to be the best parameter to characterize the transitions from SCP to DDC regimes for the $65 \%$ glycerol mixture. Figure 9 summarizes the different transitions taking place in the BTCF, based on the control parameter $\alpha$. The defect mediated turbulence (DMT) includes the SCP, the IDC and the DDC regimes. The critical value of $\alpha_{\text {def }}$ above which the first 
(a)

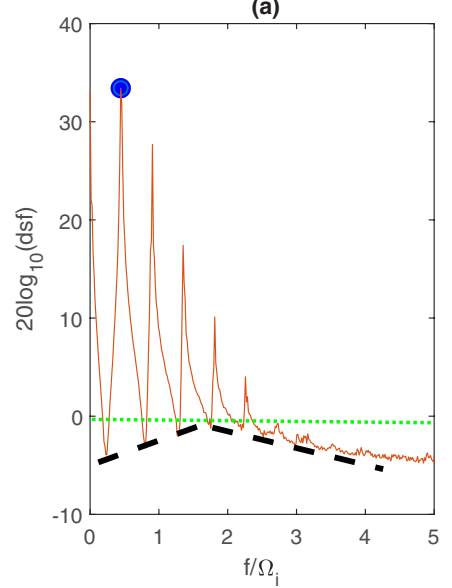

(b)

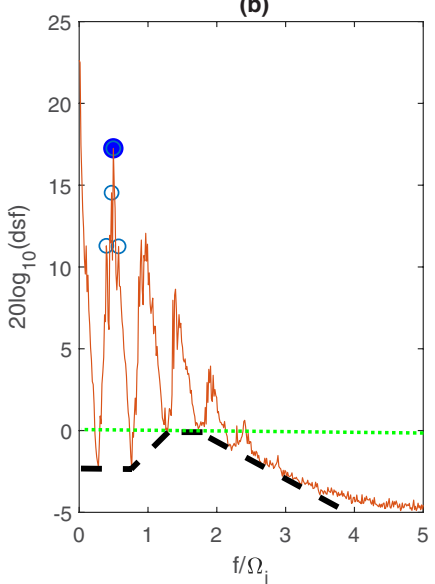

(c)

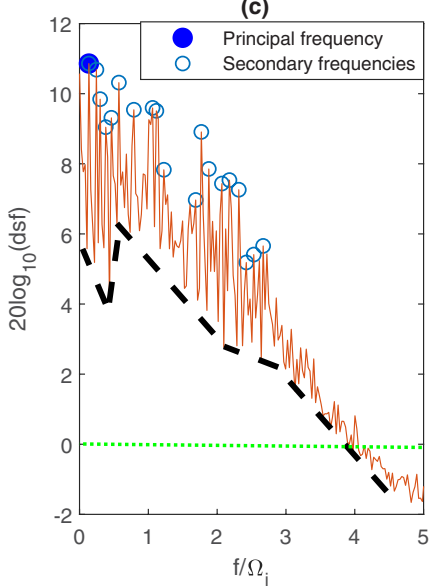

FIG. 10. Power spectrum versus frequency for: the mixutre of $65 \%$ glycerol (a) structural composite pattern $[\alpha(\%)=0.001]$, (b) intermittency defect chaos $[\alpha(\%)=0.006]$, and (c) developed defect chaos $[\alpha(\%)=0.067]$. The dashed and dotted lines are, respectively, the background noise and zero energy.

defect appears will be characterized further. $\alpha_{\text {def }}$ is also the critical volumetric fraction beyond which the flow shifts from a unique pattern regime to a composite pattern regime.

\section{B. Evolution of the frequency and wavelength with the control parameter $\alpha$ or $\operatorname{Re}$}

Frequency and wavelength are obtained by the calculations of the two-dimensional FFT from the space-time diagrams. In the frequency domain spectrum (Fig. 10), we localize the principal frequency $f_{p}$ at the maximum of the energy peak. While the secondary frequencies are defined at the energy peaks that satisfy the two following conditions:

(1) The prominence is larger than $2 d B$. The prominence is defined as energy difference between the value of the energy at the peak and its background noise (the dashed line in Fig. 10).

(2) The background noise is larger than zero energy (dotted line in Fig. 10). Even if the prominence is larger than $2 d B$ but the background is smaller than zero, the frequencies are not counted among the secondary frequencies as the cases of the harmonics [Fig. 10(a)].

Three examples of the frequency spectrum are given in the Fig. 10 that correspond to SCP [Fig. 10(a)], IDC [Fig. 10(b)], and DDC [Fig. 10(c)]. The background noise and the number of the secondary frequencies increase as the control parameter $\alpha$ is increased in the IDC and in DDC regimes [Figs. 10(b) and 10(c)].

The characteristic frequencies are plotted as a function of $\alpha$ in Fig. 11 for the $40 \%$ and $65 \%$ glycerol mixtures. For the different flow regimes, the principal frequency has a constant value around $f_{p} \approx \Omega_{i} / 2$, except for four cases in the $40 \%$ glycerol mixtures where $f_{p} \approx \Omega_{i}$. As explained by Murai et al. [8], the bubbles patterns are expected to propagate azimuthally in time with a phase velocity of half the inner cylinder tangential velocity $\left(V_{i}\right)$ and with a wave number of 1 . This is in agreement with what is observed at the occurrence of the azimuthal wave in a single-phase Taylor-Couette flow with rotating inner cylinder and steady outer cylinder (occurrence of the third instability). In the single-phase flow, the occurrence of the third instability is systematically characterized by an angular phase velocity of the azimutal wave of $0.5 \Omega_{i}$ [35] and by an azimuthal wave number of 1 for large aspect ratio $\Gamma$ [36]. The difference with the single-phase flow here is that for the Reynolds numbers range of the study, the azimuthal wave does not exist in the single-phase flow, it occurs due to the bubbles.

Our interpretation is that this azimuthal phase velocity of $0.5 V_{i}$ is in agreement with a preferential bubble capture in the bulk flow inside the Taylor vortices. In the case when the azimuthal wave 


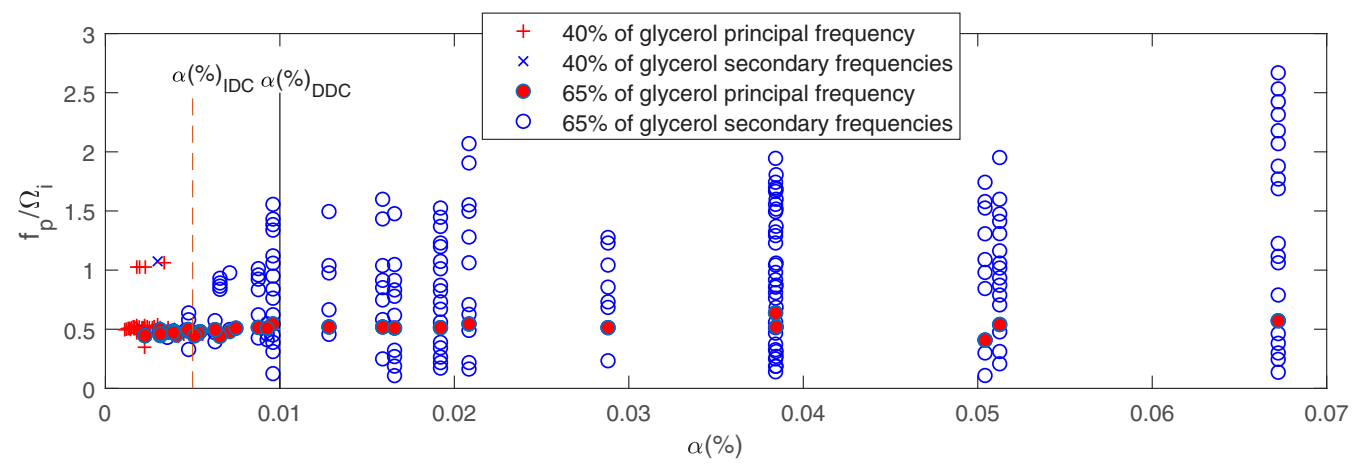

FIG. 11. Evolution of the characteristic frequencies normalized by the inner cylinder angular velocity as a function of $\alpha$ (red, principal frequency; blue, secondary frequencies).

frequency is $f_{p} \approx \Omega_{i}$, this correspond to operating points at high Reynolds number in the $40 \%$ glycerol mixture, where the flow regime is a unique flow pattern of wavy spiral or a structured composite flow (SCP) of preferential wavy spiral. In this case, the azimuthal wave number is 2 and the frequency of the helicoidal path is $0.5 \Omega_{i}$. For $\alpha<\alpha_{\mathrm{IDC}}$, for the unique pattern flow or the structured composite patterns flow, the power spectrum depicts a single peak of wavy frequency $f_{p}$. For $\alpha_{\mathrm{IDC}} \leqslant \alpha \leqslant \alpha_{\mathrm{DDC}}$, in the intermittency defect chaos regime, the power spectrum has several peaks around the principal frequency $f_{p}$. In this case, the secondary characteristic frequencies vary between $0.4 \Omega_{i}$ and $\Omega_{i}$. The presence of these several peaks around the principal frequency $0.5 \Omega_{i}$ is due to the coexistence of different basic patterns in the composite flows with defects. For large values of $\alpha$, the power spectrum is a large bandwidth spectrum in the developed defects chaos regime.

The evolution of the axial wavelength is plotted with regard to the Reynolds number (Fig. 12) and with regard to the volumetric air fraction $\alpha$ (Fig. 13). The axial wavelength increases slowly with Re number from $2.8 d \pm 0.2 d$ to $3.5 d \pm 0.2 d$, same trend is encountered for our geometry of Taylor-Couette device in single-phase flow [9]. The axial wavelength is smaller in the bubbly flow than in the single-phase flow, as expected from Fokoua et al. [9] for a preferential capture of the bubbles in the vortices. The axial wavelength decreases with respect to $\alpha$ from $3.6 d$ to $2.5 d$ and it remains constant for large $\alpha$.

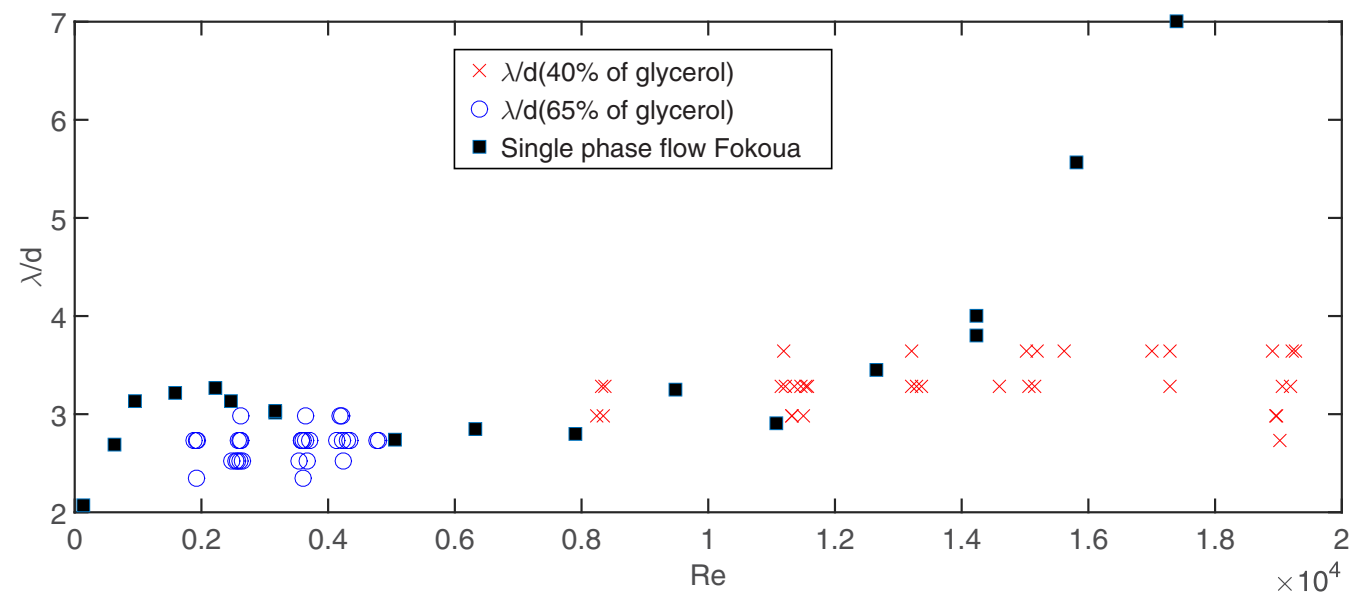

FIG. 12. Evolution of the normalized axial wavelength as a function of Re number. 


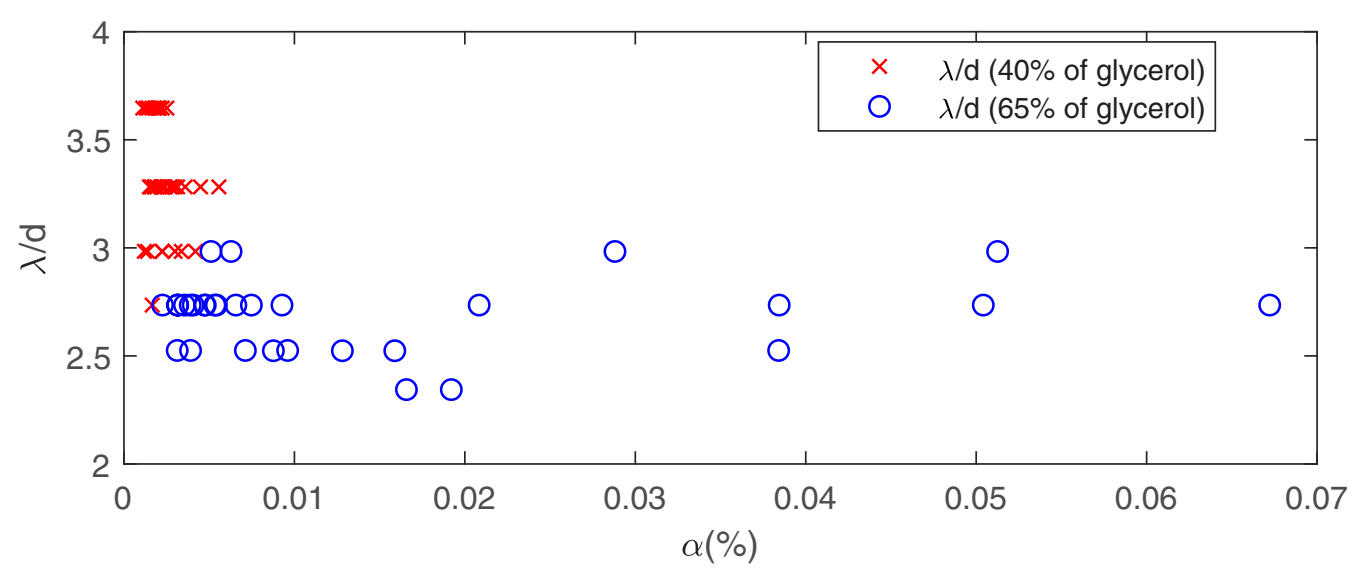

FIG. 13. Evolution of the normalized axial wavelength as a function of $\alpha$ control parameter.

\section{Evolution of the correlation time and correlation length with $\alpha$}

The patterns observed in our experiments are analyzed by the calculation of the space-time correlation function as described in Ref. [37]:

$$
\operatorname{Cor}(\Delta z, \Delta t)=\frac{\langle\widetilde{I}(z+\Delta z, t+\Delta t) \widetilde{I}(z, t)\rangle}{\left\langle\widetilde{I}(z, t)^{2}\right\rangle},
$$

where $\widetilde{I}(z, t)=I(z, t)-\langle I(z, t)\rangle$. The analysis of the space-time correlation functions [Eq. (10)] permits to characterize the transitions inside the DMT regimes. Indeed, in the DMT regime, the correlation function is expected to vary exponentially with time and distance [Eq. (11)] in agreement with the numerical simulations of the complex Ginzburg-Landau equation (CGLE) performed by Shraiman et al. [30]:

$$
\operatorname{Cor}(\Delta z, \Delta t) \sim \exp (-\Delta t / \tau) \exp (-\Delta z / \xi)
$$

$\tau$ and $\xi$ are, respectively, the correlation time and length of the pattern. To extract $\tau$ and $\xi$, the experimental correlation functions in time $\operatorname{Cor}(0, \Delta t)[$ Fig. 14(a)] and in space $\operatorname{Cor}(\Delta z, 0)$

(a)

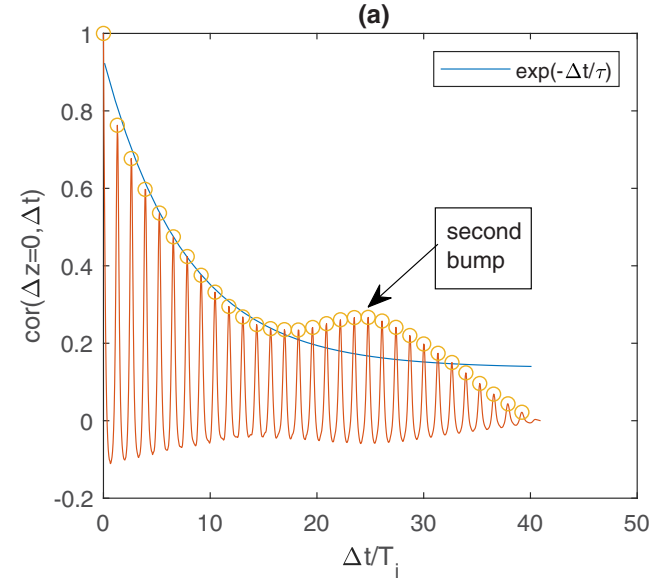

(b)

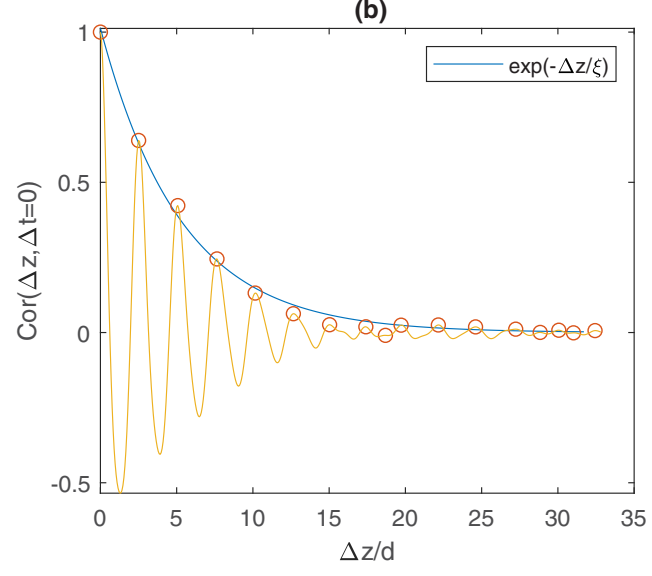

FIG. 14. (a) Time correlation and (b) length correlation and their best exponential fit for $\mathrm{Re}=3530$; $\operatorname{Re}_{g}=0.32 ; Q_{g}=30 \mathrm{ml} / \mathrm{min} ; \alpha=0.0013 \%, 65 \%$ glycerol mixture. 


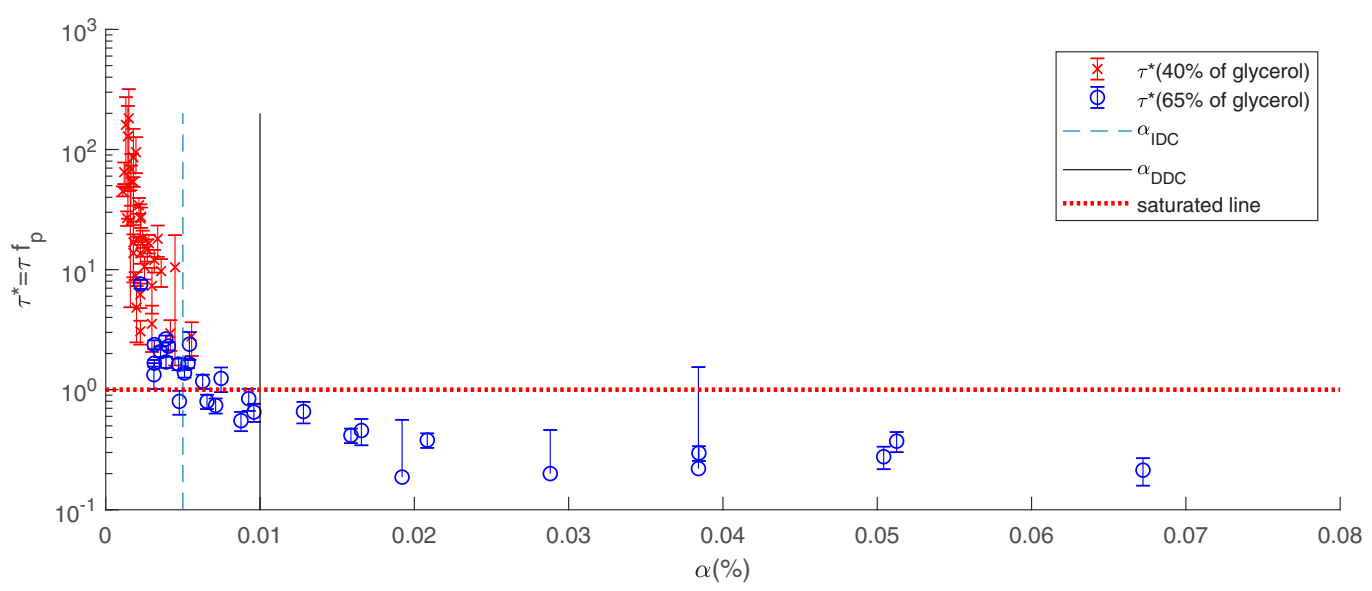

FIG. 15. Dimensional correlation time as a function of the control parameter $\alpha$ for mixtures of $65 \%$ and $40 \%$ glycerol.

[Fig. 14(b)] are fitted by the exponential functions $\exp (-\Delta t / \tau)$ for time and $\exp (-\Delta z / \xi)$ for space, respectively.

The time correlation function can exhibit a second bump as observed in Fig. 14(a) at $\Delta t / T_{i}=25$. The occurrence of this second bump in the time correlation function corresponds to the switching between two basic patterns (toroidal for $0<\Delta t / T_{i}<25$ and spiral for $25<\Delta t / T_{i}<40$ ). In this case, the exponential fit of the time correlation function is applied to the pattern that has the largest duration [toroidal for the example of the Fig. 14(a)].

The correlation time and length are scaled by the principal frequency $f_{p}$ and the wavelength of the pattern:

$$
\begin{gathered}
\tau^{*}=\tau f_{p}, \\
\xi^{*}=\xi / \lambda .
\end{gathered}
$$

The normalized correlation time $\tau^{*}$ and length $\xi^{*}$ are presented in the Figs. 15 and 16 with regard to the control parameter $\alpha$ for both mixtures of glycerol. For $\alpha(\%)<\alpha_{\text {IDC }}=0.005 \%$, the

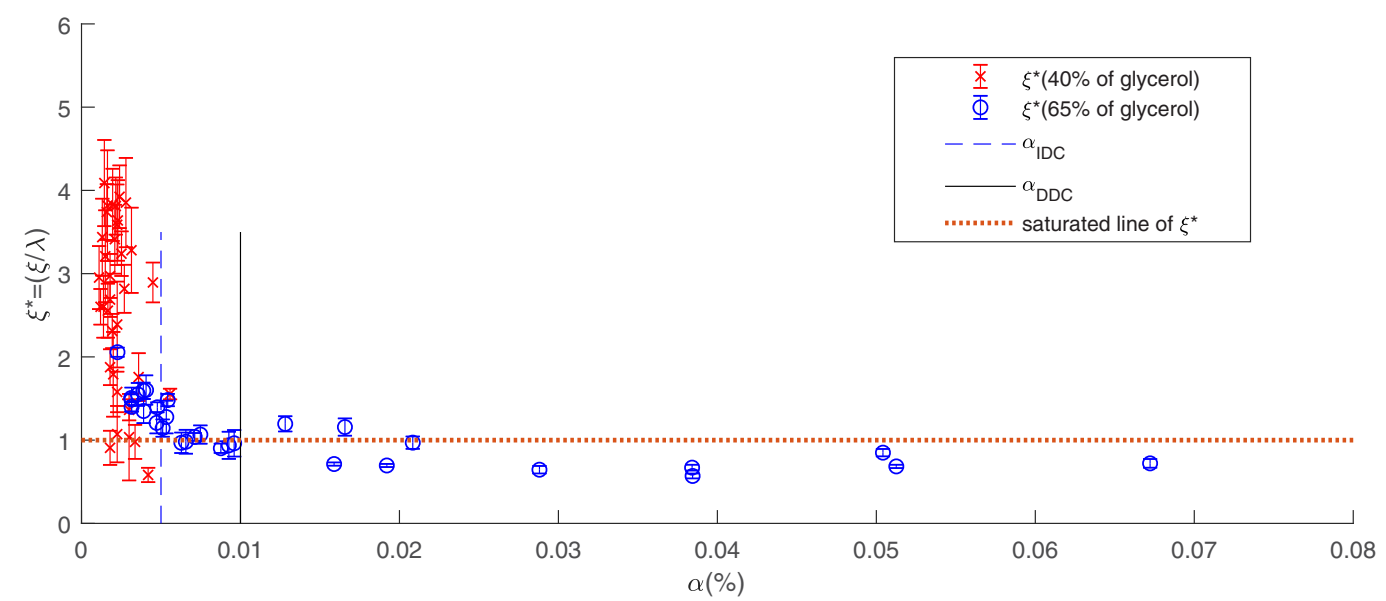

FIG. 16. Dimensionless axial correlation length as a function of control parameter $\alpha$ for $65 \%$ and $40 \%$ mixture. 


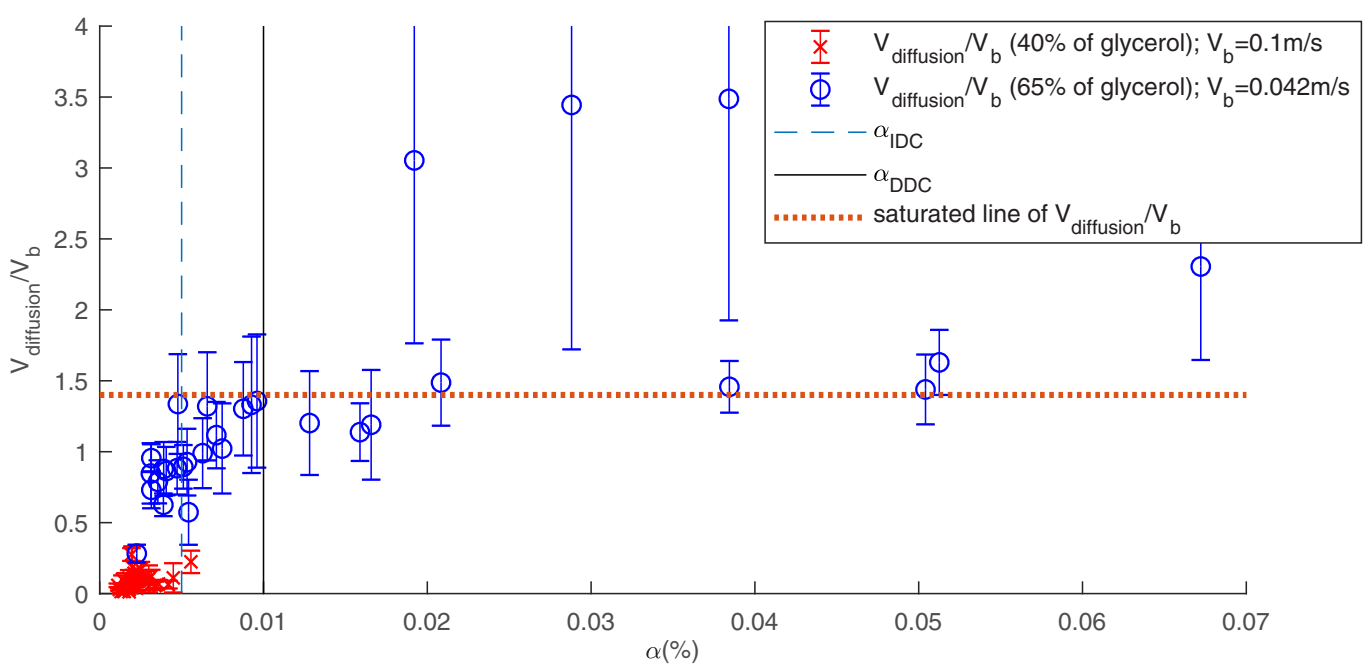

FIG. 17. Evolution of the axial diffusion velocity scaled by bubbles velocity $V_{b}$ with the parameter $\alpha(\%)$.

normalized correlation time and length decrease strongly with $\alpha$ from 200 to 1 for $\tau^{*}$ and from 4 to 1 for $\xi^{*}$. In this regime, the increase of defects number is responsible for this strong decrease of spacetime correlation in the pattern. Meanwhile, the flow has the capacity to reproduce the pattern in axial direction and in time; the correlation length and time remain larger than the space-time period of the flow $\left(\xi^{*}>1\right.$ and $\left.\tau^{*}>1\right)$. As mentioned before, the flow is structured, as it keeps the memory of the pattern. For $\alpha(\%) \geqslant \alpha_{\mathrm{IDC}}=0.005 \%$, the bubbly patterns have small correlations, the correlation length and time being smaller than the space-time periods $\left(\xi^{*}<1\right.$ and $\left.\tau^{*}<1\right)$. This critical value of the air volumetric fraction $\alpha_{\mathrm{IDC}}=0.005 \%$ correctly characterizes the transition from the structure patterns flow (SCP) to the intermittent defect chaos regime (IDC). In the DDC regime, for $\alpha>0.02 \%$, the normalized correlation length and time saturate. This means that the dynamics of the defects does not change anymore. Indeed, the defects number is expected to saturate, as the gap is of finite dimension. We can suppose that saturation occurs at large alpha when a connection between Taylor vortex pairs (defect) occurs over each time and axial periods. The azimuthal wave promotes the connection by making easier bubbles jumping from the crest to the trough of the upper Taylor vortex pair. Bubbles jumping (i.e. bubbles escape from the vortices) is enhanced (1) by the increase in the gravity effect (achieved by increasing the relative contribution of the terminal rising velocity of isolated bubbles $V_{b} / V_{i}$ or decreasing $V_{i}$ and (2) by an important accumulation of bubbles in the Taylor vortices (achieved at large values of $Q_{g}$ ) which induces an important rising velocity of bubbles clouds under collective effect An important axial flux of the bubbles is required to connect the vortex pairs by bubbles jumping: this is ensured at large values of the volumetric fraction $\alpha \sim Q_{g} / V_{i}$.

From Fig. 16, at saturation, we note $\frac{\xi}{\lambda} \approx 0.6$. This value is quite in agreement with the axial distance $\frac{d_{2}}{\lambda} \approx 0.56$ measured by Fokoua et al. [9] between bubbles rings trapped in the counterclockwise and clockwise vortices. Thus, we can conclude that the axial position of bubbles trapping in the vortices at equilibrium controls the axial distance of the bubbles jumping and that this is the systematic bubbles jumping at high volumetric fraction that controls the correlation properties of the patterns at saturation.

The axial diffusion rate of the bubbly patterns is defined as

$$
V_{\text {diffusion }}=\frac{\xi}{\tau} \text {. }
$$

Figure 17 shows the evolution according to $\alpha$ of the axial diffusion rate scaled by the terminal rising bubble velocity $V_{b}$ (values given in Table I) For the $65 \%$ glycerol mixture, it evidences 
different scaling laws of the diffusion rate with respect to the regime. For $\alpha<\alpha_{\mathrm{DDC}}=0.01 \%$, in the SCP and IDC regimes, the diffusion rate expands linearly with $\alpha$. According to Kowe et al. [38], the volume of a bubbly dispersed flow can be modelized using a three-fluids model. It is composed of a volume of bubbles of effective volume fraction $\alpha_{v}$, a volume of liquid advected by the bubbles and a volume of undisturbed liquid of velocity $V_{0}$. In this framework, considering $C_{a}$, the added mass of bubbles $\left(C_{a} \approx 0.5\right)$, the axial velocity of the liquid in the two-phase flow $V_{z}$ can be approximated by the following equation:

$$
\left(1-\alpha_{v}\right) V_{z}=\left(1-\alpha_{v}-\alpha_{v} C_{a}\right) V_{z 0}+\alpha_{v} C_{a} V_{b}=\left(1-\alpha_{v}\right) V_{z 0}+\alpha_{v} C_{a}\left(V_{b}-V_{z 0}\right) .
$$

As the basic Taylor-Couette single-phase flow is characterized by no axial mean velocity of the flow, it yields

$$
V_{z} \approx\left(1-\alpha_{v}\right) V_{z}=\alpha_{v} C_{a} V_{b}
$$

The axial diffusion of the bubbly patterns is linked to the axial velocity of the liquid induced by the bubbles $V_{z}$. Assuming that the effective air volume fraction $\alpha_{v}$ evolves linearly with the air volumetric fraction $\alpha$, we obtain a linear scaling law of $V_{\text {diffusion }} / V_{b}$ with regard to $\alpha: V_{\text {diffusion }} \simeq$ $V_{z} \sim \alpha V_{b}$.

Interesting enough is the fact that the critical value of the air volumetric fraction $\alpha_{\mathrm{IDC}}=0.005 \%$, characteristic of the transition from the SCP regime to IDC regime is also the critical value of alpha which leads to equality between the diffusion rate and the bubble terminal rising velocity. In the DDC regime, for $\alpha \geqslant \alpha_{\mathrm{DDC}}=0.01 \%$, the patterns have a diffusion rate larger than the bubbles rising velocity. At saturation, the diffusion velocity is in agreement with the value of the bubbles jumping velocity. Collective effects of the jumping can increase the effective rising velocity of bubbles clusters (up to $1.5 V_{b}$ ). This confirms the fact, that bubbles release from the Taylor vortices and jumping at each time period and axial period is the mechanism that controls the diffusion rate at saturation in the DDC regime. The axial diffusion rate is representative of the velocity at which the defects will propagate axially. For all regimes, this traveling velocity of the defects obviously increases with the terminal rising velocity of bubbles, which implies that a change in the bubble size or viscosity would modify the dynamics of the defects under gravity effects. Next parts are devoted to the characterization of the defects.

\section{Evolution of the number of defect with $\alpha$}

All the spatiotemporal patterns that contain defects are analyzed using the complex demodulation technique by Hilbert Transform to extract the module $|A(z, t)|$ and the phase $\Phi(z, t)$ [39]. The space-time diagram is transformed into its complex quantity as

$$
I(z, t)=\Re\{\widehat{I}(z, t)\}=\Re\{|A(z, t)| \exp (i \Phi(z, t))\},
$$

where $\Re$ is the real part of the complex signal $\widehat{I}(z, t)$ obtained by the complex demodulation. To realize the complex demodulation, first, the two-dimensional spectrum is calculated from the real signal $I(z, t)$ by the Fourier transforms $\widehat{I}(k, f)$ to detect the most energetic peak of the pattern. Then, the Fourier transform $\widehat{I}(k, f)$ is filtered $\left[\widehat{I}_{\mathrm{GF}}(k, f)\right]$ using the two-dimensional Gaussian filter (GF), $\exp \left[-\left(f-f_{p}\right)^{2} /\left(2 \sigma_{f}\right)\right] \exp \left[-\left(k-k_{p}\right)^{2} /\left(2 \sigma_{k}\right)\right]$, where $f, k$ and their standard deviations $\sigma_{f}, \sigma_{k}$ are chosen to keep the energetic modes: $k \in\left[0.5 k_{p}, 1.5 k_{p}\right]$ and $f \in\left[0.01 f_{p}, 5 f_{p}\right]$. Afterwards, from the filtered Fourier transform $\widehat{I}_{\mathrm{GF}}(k, f)$, the Fourier inverse transform allows to recover the complex quantity of the signal $\widehat{I}(z, t)=|A(z, t)| \exp [i \Phi(z, t)]$, from which the space-time diagrams of the module $|A(z, t)|$ and phase $\Phi(z, t)$ are obtained, respectively.

An example of the result of the complex demodulation technique is shown in Figs. 18(a) $-18(\mathrm{e})$. Comparison of space-time diagrams of the module and phase shows the existence of two defects of opposite charges: $v_{+}=+1$ for positive defect when the phase presents a positive discontinuity $(+2 \pi)$ and $\nu_{-}=-1$ for negative defect when the phase presents a negative discontinuity $(-2 \pi)$. In the vicinity of the defect, the module decreases strongly and vanishes in its core [Fig. 18(d-1)]. We 


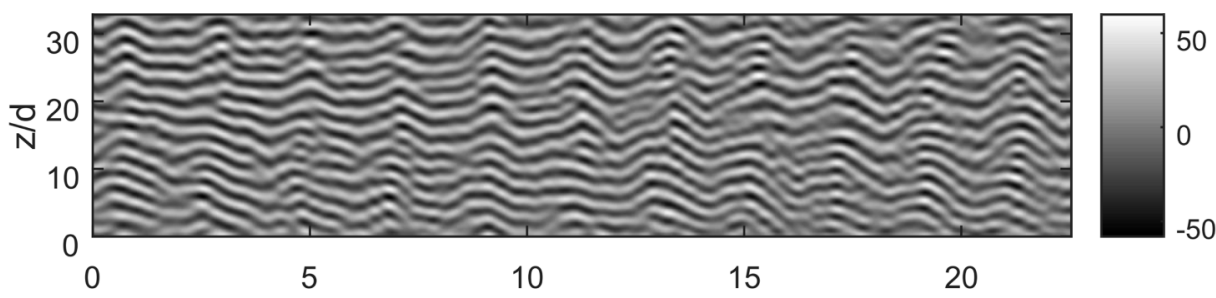

(a) $t / \mathrm{T}_{\mathrm{i}}$
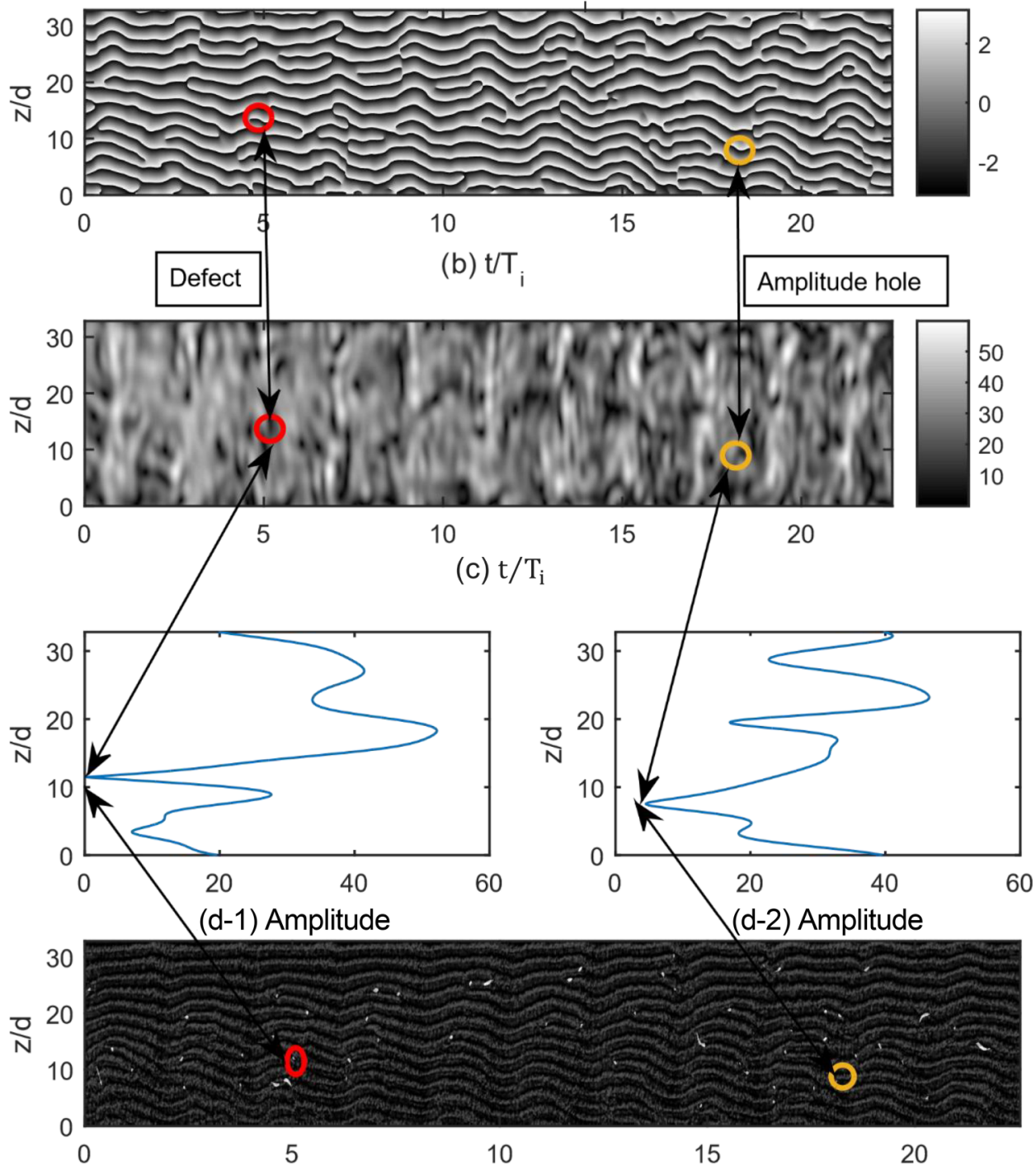

(e) $t / T_{i}$

FIG. 18. Complex demodulation for $\left(\operatorname{Re}=2648 ; Q_{g}=160 \mathrm{ml} / \mathrm{min} ; \alpha=0.0087 \% ; \operatorname{Re}_{g}=0.16 ; 65 \%\right.$ of glycerol mixture: (a) space-time diagram of analytical prolongment, (b) space-time diagram of phase, (c) module, (d-1) axial profile of the module near a defect $\left(t=5.1 T_{i} ; z=11 d\right),(\mathrm{d}-2)$ axial profile of the module near a hole $\left(t=18.1 T_{i} ; z=9 d\right)$, (e) cartography of binarized module (defects in white) superimposed with the cartography of phase. 


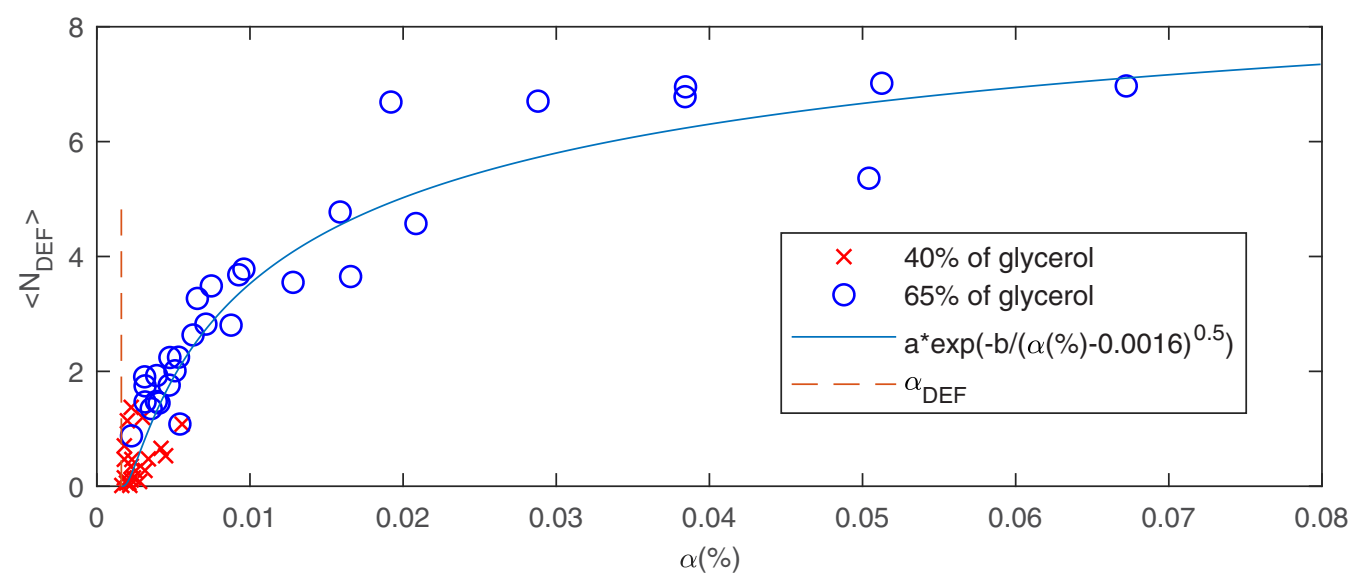

FIG. 19. Evolution of the average number of defects according to the parameter $\alpha$ for mixtures $65 \%$ and $40 \%$ of glycerol, the solid line represents the law of adjustment proposed by Egolf and Greenside [31] [Eq. (19)] for the mixture of $65 \%$ glycerol.

observe that the defects are associated with strong modules variation of the patterns [Fig. 18(c)]. The defects with absolute annihilation of the module are discriminated from the holes observed in the bubbles patterns. Indeed, in the holes, the module reaches a minimum, that is larger than zero [Fig. 18(d-2)]. In the presence of defects, the number of vortices of the liquid phase change: the vortices can merge or split. For this case, the amplitude is equal to zero and the phase of the wave presents a discontinuity of the phase $( \pm 2 \pi)$. The physical meaning of these defects is the breaking of the phase of the vortices accompanied by the local change of the wavelength [Figs. 18(a) and 18(b)]. The axial wavelength increases when the vortices merge and decreases when the vortices have a dislocation [Figs. 18(a) and 18(b)]. The variation of the wavelength is the result of an instability of the phase known as Benjamin-Feir instability [19]. The dark regions in the space-time cartography of the module [Fig. 18(c)] with absolute annihilation of the module correspond to defects. To detect the defects in the space-time diagram, we have binarized the cartography of the module [Fig. 18(c)]: the defects correspond to amplitude equal zero (white color: $|A|=0$ ) and pattern without defects correspond to amplitude larger than zero (black color: $|A|>0$ ). In Fig. 18(e), we present a superposition of the cartographies of both phase and binarized module relative to defect detection.

From the binarized module [Fig. 18(e)], we can count the number $N_{\mathrm{DEF}}$ of defects during a whole experimental run and determine the mean number of defects as

$$
\left\langle N_{\mathrm{DEF}}\right\rangle=N_{\mathrm{DEF}} / \tilde{T}_{\mathrm{acq}}=\left(N_{\mathrm{DEF}} T_{i}\right) / T_{\mathrm{acq}},
$$

where $\tilde{T}_{\text {acq }}=T_{\text {acq }} / T_{i}$ is the dimensionless duration of data acquisition. The variation of $\left\langle N_{\mathrm{DEF}}\right\rangle$ with the control parameter $\alpha$ is plotted in Fig. 19. The number of defects increases with the control parameter $\alpha$ for $\alpha<0.02 \%$ before saturation occurs. The defect dynamic observed in our study is similar of that observed in the numerical simulations of the complex Ginzburg-Landau equation [30,31]. In the theory of the DMT, the number of defects can increases with $c_{3}$ following the equation 19 suggested by Egolf and Greenside [31], where $c_{3}$ is the nonlinear dispersion coefficient in the Ginzburg-Landau equation [Eq. (1)]. $c_{3}^{\prime \prime}$ is the critical value of $c_{3}$ at the threshold of the DMT; $a, b$ and $p$ are free parameters used to fit the numerical data of the number of defects [31] with Eq. (19). In the experimental investigation of the DMT, Cros and Le Gal [25] and Latrache et al. [29] have used Eq. (1) to fit the variation of the number of defects with $c_{3}$, in their experiments, $c_{3}$ is the Reynolds number or Taylor number Ta $=\sqrt{\frac{d}{R_{i}}}$ Re by taking the curvature of the Taylor-Couette system for the case of viscoelastic flow [29]. In our study, the number of defects is fitted by 
TABLE II. Eglof and Greenside [31] fitting parameter.

\begin{tabular}{lccccc}
\hline \hline Fitting parameters of Eq. (19) & & $a$ & $b$ & $c_{3}^{\prime \prime}$ & $p$ \\
\hline Equation of Ginzburg-Landau [31] & 0.66 & 0.98 & 0.70 & 1 \\
Torsional Couette flow [25] & 5 & 4 & 38.5 & 1 \\
Viscoelastic Taylor-Couette [29] & $E=0.011$ & $0.058 \pm 0.004$ & $0.25 \pm 0.04$ & 49.8 & 1 \\
& $E=0.020$ & $0.008 \pm 0.001$ & $0.86 \pm 0.05$ & 46.6 & 1 \\
& $E=0.046$ & $0.032 \pm 0.001$ & $6 \pm 0.08$ & 43.5 & 1 \\
Present study: bubbly Taylor-Couette flow & $0.5 \pm 0.2$ & $0.10 \pm 0.05$ & $\alpha_{\text {DEF }}(\%)=$ & $0.5 \pm 0.05$ \\
& & & & $0.0016 \pm 0.0002$ & \\
\hline \hline
\end{tabular}

the Eq. (19) when the control parameter of the DMT $c_{3}$ considered is the air volumetric fraction $\alpha=c_{3}$ :

$$
\left\langle N_{\mathrm{DEF}}\right\rangle=a \exp \left[-b /\left(c_{3}-c_{3}^{\prime \prime}\right)^{p}\right] .
$$

The best fit coefficients of Eq. (19) obtained for our experimental results of the $65 \%$ glycerol mixture yields the values of $a, b, p$, and $c_{3}^{\prime \prime}$ given in Table II; $\alpha$ plays the same role as $c_{3}$ and $c_{3}^{\prime \prime}=$ $\alpha_{\text {def }}=0.0016 \%$ is the critical value of the air volumetric fraction above which the defect mediated turbulence regime DMT takes place. In Table II, we summarize also the coefficients obtained in the previous work for the DMT observed in different systems.

In the DDC regime for $\alpha>0.02 \%$, it is confirmed that the number of defects saturates (Fig. 19) as expected for a device of finite dimensions. $\frac{N_{\text {def }}}{T_{a c q} f_{p}} \approx 14$ is representative of the number of defects per time period of the bubbles patterns over the entire height of the device, it is quite equal to $L / \lambda$, which confirms that at saturation, we have one defect over each axial period of the Taylor vortices and over each time period of the azimuthal wavelength.

\section{E. Spatiotemporal properties in the transitions of the DMT regimes}

The separation time between two consecutive defects $T_{\text {sep }}$ and the lifetime $T_{\text {def }}$ of each defect are determined, based on the cartographies of the binarized module of the space-time diagrams complex demodulation [Fig. 18(e)]. The averaged lifetime $\left\langle T_{\text {def }}\right\rangle$ and the averaged separation time $\left\langle T_{\text {sep }}\right\rangle$ are the arithmetic average among all the defects of a space-time diagram. Figures 20 and 21 display $\left\langle T_{\mathrm{def}}\right\rangle$ and $\left\langle T_{\mathrm{sep}}\right\rangle$ scaled by the rotation period Ti as a function of the control parameter $\alpha$. For the

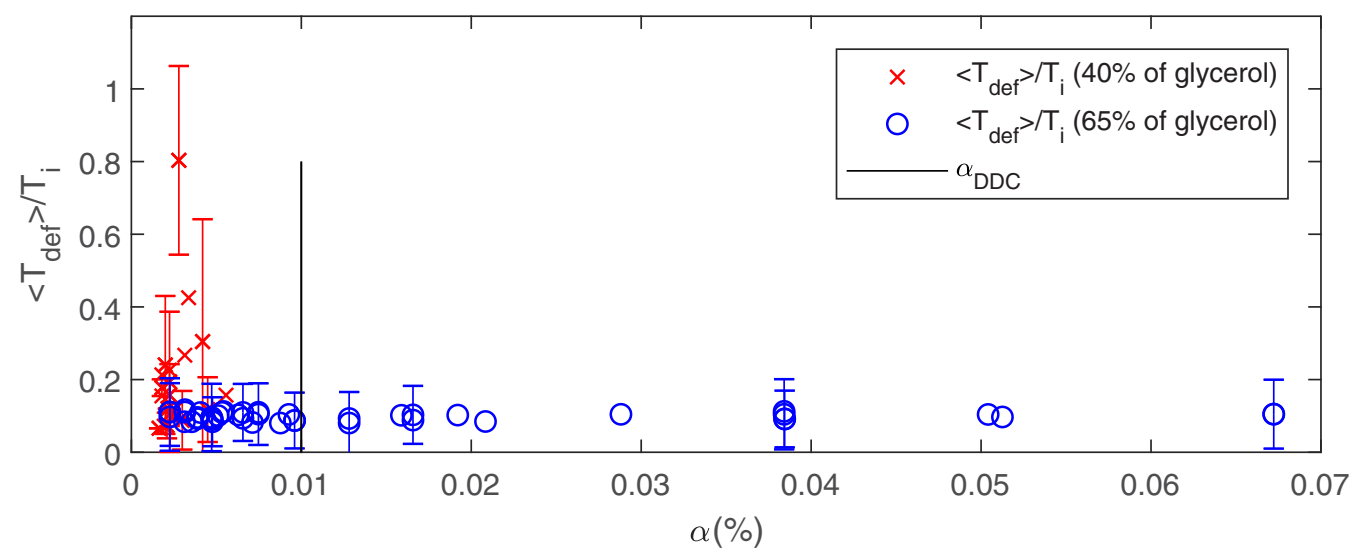

FIG. 20. Normalized averaged lifetime of the defects according to $\alpha$ for mixtures of $65 \%$ and $40 \%$ glycerol. Error bars represent standard deviations. 


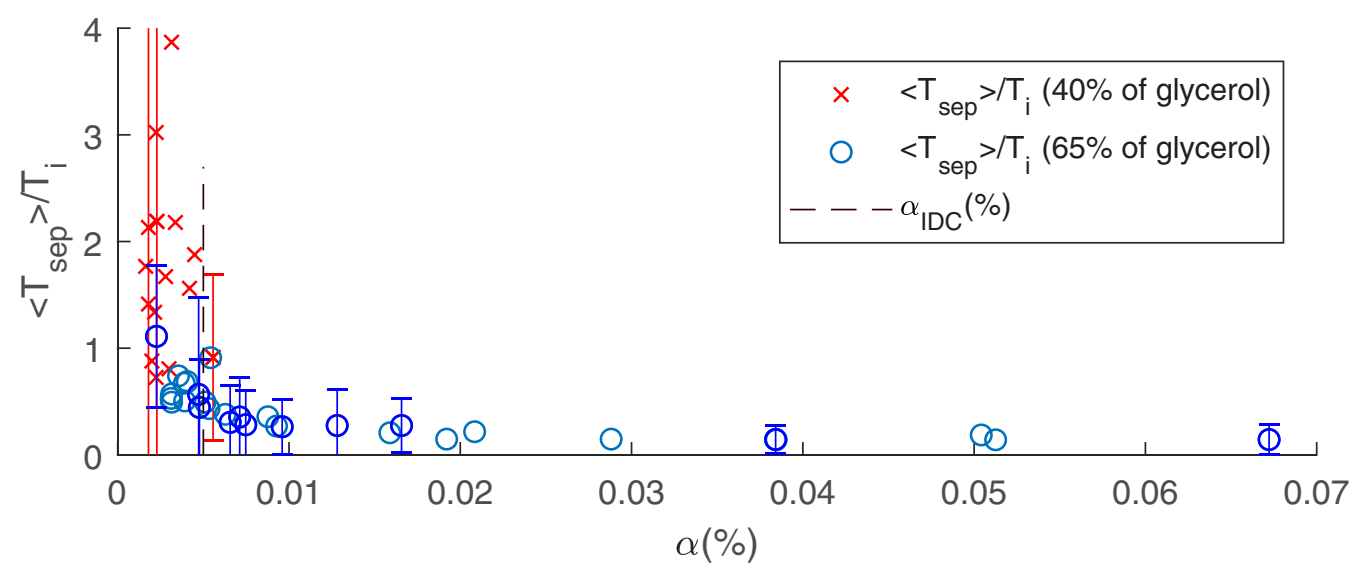

FIG. 21. Normalized time separation between two consecutive defects according to $\alpha$ for mixtures of $65 \%$ and $40 \%$ glycerol. Error bars represent standard deviations.

mixture of $65 \%$ glycerol, and for $\alpha>\alpha_{\mathrm{DDC}}=0.01 \%$, the separation time between two consecutive defects is of the same order as the lifetime of defect: $\left\langle T_{\text {def }}\right\rangle \approx\left\langle T_{\text {sep }}\right\rangle \approx 0.1 T_{i}$. This result has been reported in the theoretical calculation of Afraimovich and Burnimovich [40] and is characteristic of the DDC regime. As can be seen in Fig. 21, for the mixture of $65 \%$ glycerol, and for $\alpha>\alpha_{\mathrm{IDC}}=$ $0.005 \%$, the standard deviation of the separation time $\sigma_{T_{\text {sep }}}$ is of the same order as the averaged value of the separation time $T_{\text {sep }}$. Also, for this range of $\alpha$ values, the mean number of defects is equal to the inverse of the averaged separation time: $\left\langle N_{\mathrm{DEF}}\right\rangle=T_{i} /\left\langle T_{\mathrm{sep}}\right\rangle$, as confirmed by Fig. 22. This is in agreement with an exponential decrease of the distribution of the separation time (Fig. 23), and a Poisson distribution of the occurrence number of the defects, as observed from $[25,29]$. This result confirms that the nucleation of the defects freezes the dynamics of the patterns around the defect and validates the role of a homoclinic orbit in the transition scenario proposed by Afraimovich and Bunimovich [40].

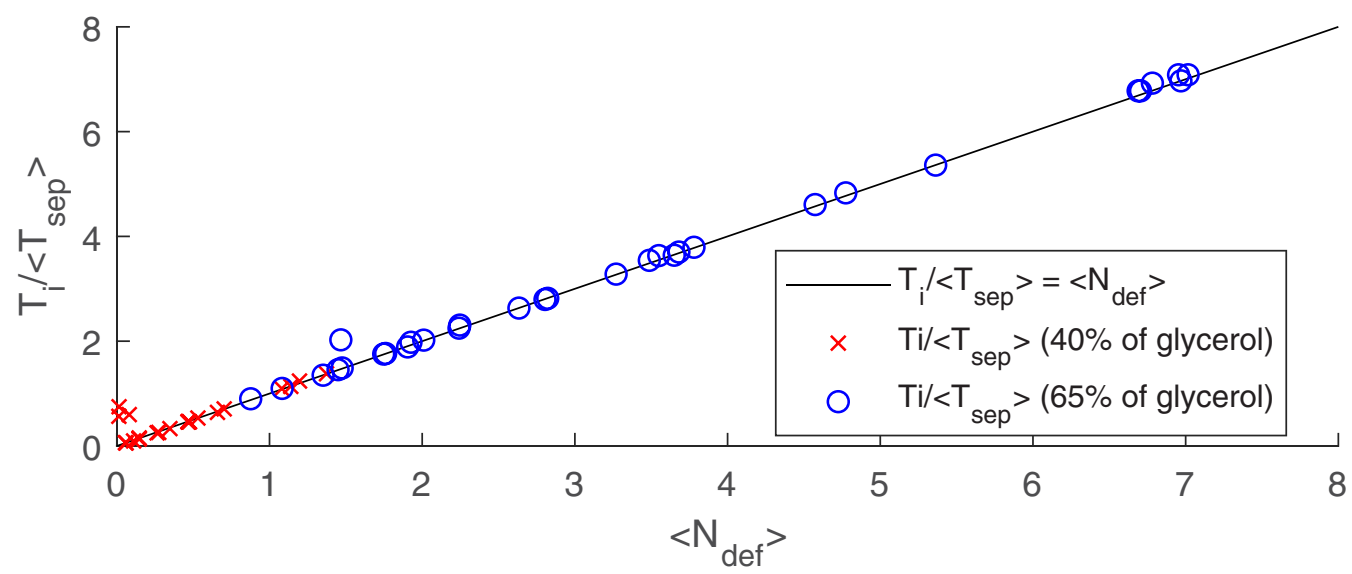

FIG. 22. Inverse of the averaged separation time in function of the mean number of defects. 


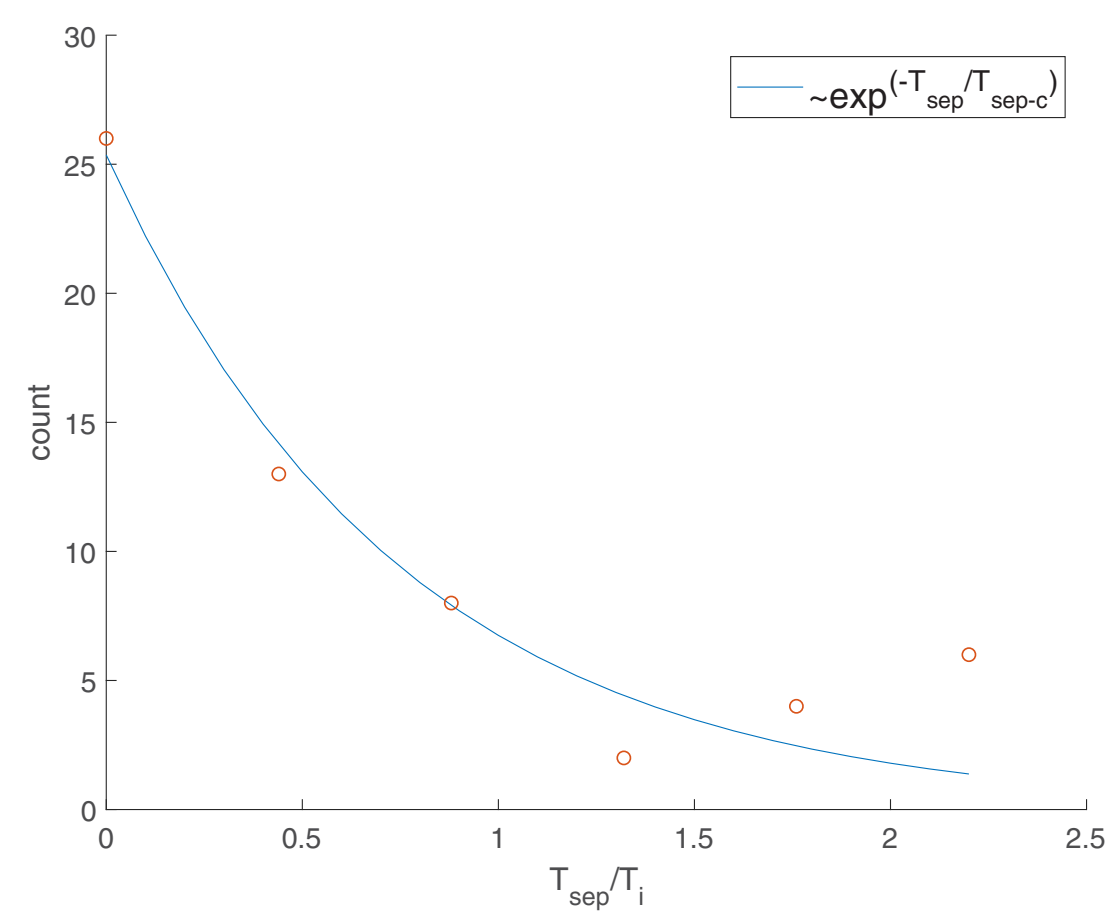

FIG. 23. Histogram of the separation time between two consecutive defects fitted by exponential law for $\operatorname{Re}=2867 ; Q_{g}=99.2 \mathrm{ml} / \mathrm{min} ; \alpha(\%)=0.0054 ;$ mixture $65 \%$ of glycerol.

The covariance of the separation time between two consecutive defects can be calculated using the following definition:

$$
C o T_{\text {sep }}=\frac{1}{N_{\text {def }}-1} \sum_{i=1}^{N_{\text {def }}-1}\left(T_{\text {sep }}^{i}-\left\langle T_{\text {sep }}\right\rangle\right)\left(T_{\text {sep }}^{i+1}-\left\langle T_{\text {sep }}\right\rangle\right) .
$$

The covariance [Eq. (20)] gives an information about the independency of the defects occurrence. For $\alpha \geqslant \alpha_{\text {IDC }}=0.005 \%$, the covariance (Fig. 24) is almost equal to zero $\left(C o T_{\text {sep }} \approx 0\right)$, the defects

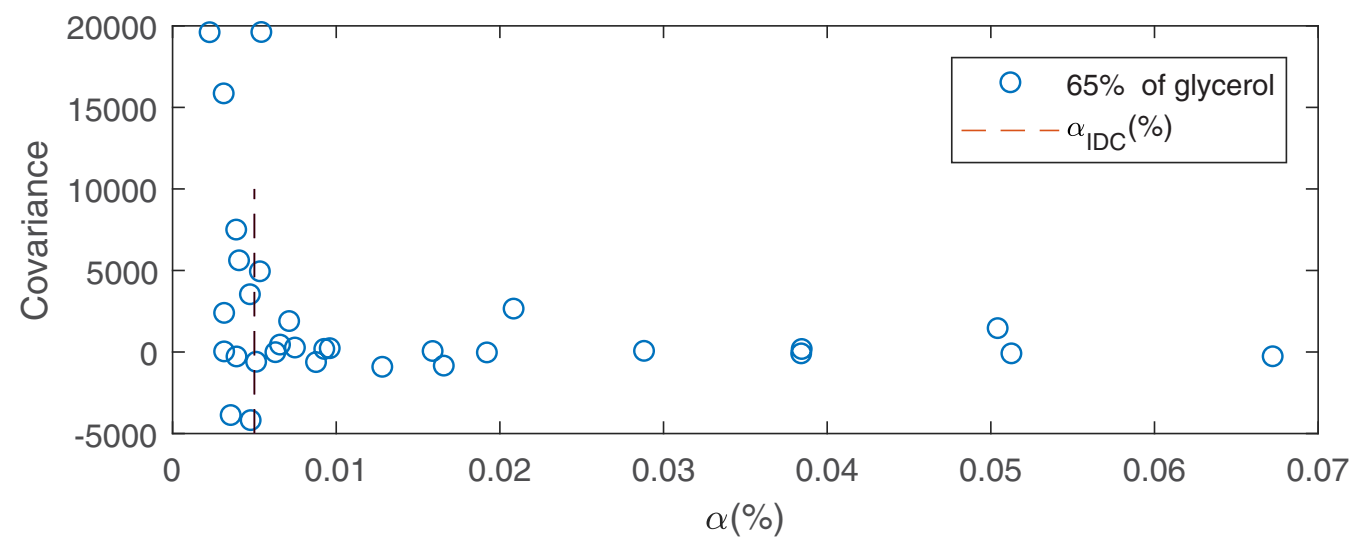

FIG. 24. Covariance of the consecutive separation times between defects as a function of $\alpha(\%)$ for the mixture of $65 \%$ glycerol. 


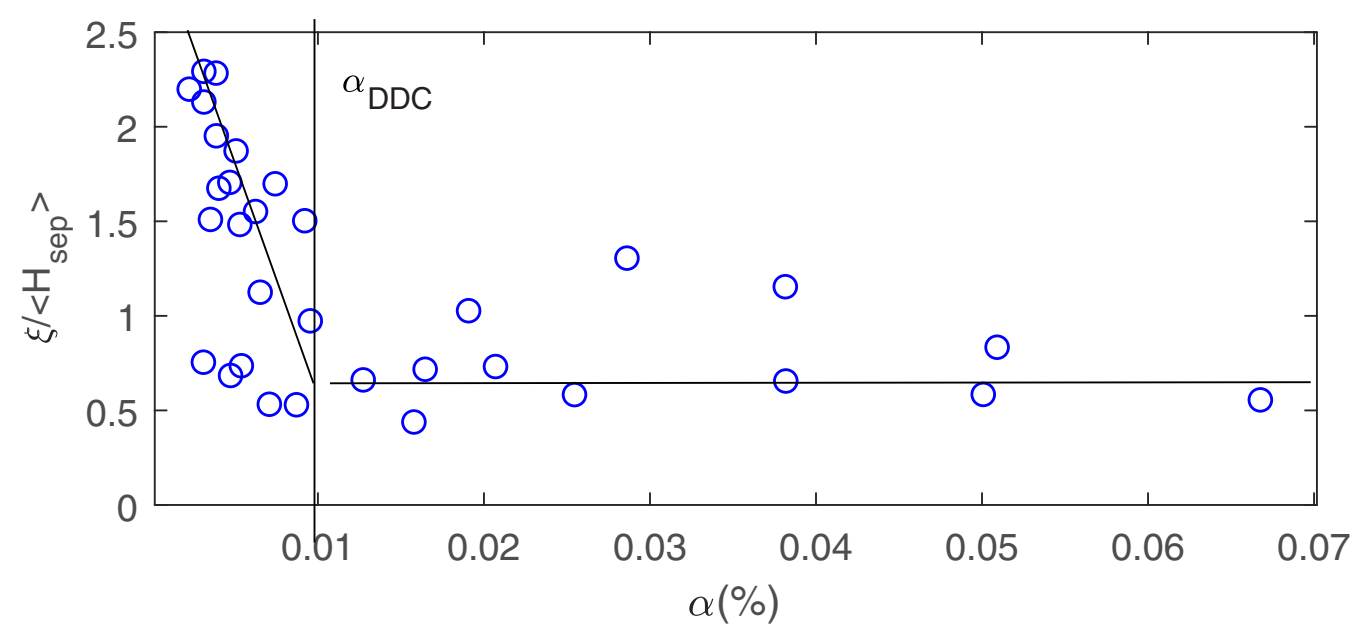

FIG. 25. Ratio of the correlation length to the averaged axial defect separation length according to $\alpha$ for the mixture of $65 \%$ glycerol.

occurrences are independent events. This is in agreement with the exponential distribution law of separation times where $\sigma_{T_{\text {sep }}}$ is of the same order as the averaged value of the separation time $\left\langle T_{\text {sep }}\right\rangle$. This critical value of $\alpha_{\text {IDC }}$ is in agreement with that obtained by the evolution of the correlation time and length when they are smaller than the spatiotemporal period.

The axial separation length between two consecutive defects $H_{\text {sep }}$ is also determined based on the cartographies of binarized module of the complex demodulation [Fig. 18(e)] and the averaged value $\left\langle H_{\text {sep }}\right\rangle$ is calculated by arithmetic mean among all defects of the space-time diagram. In the theoretical work of Coullet et al. [18], the transition to developed turbulence occurs when the correlation length of the system becomes of the same order as the averaged distance between defects [18]. By the same idea, we have plotted the ratio of correlation length to the mean axial separation length between two defects (Fig. 25). The ratio $\xi /\left\langle H_{\text {sep }}\right\rangle$ decreases from 2.5 to 1 at $\alpha=\alpha_{\mathrm{DDC}}=0.01 \%$ which corresponds to the transition to the developed defects chaos regime (DDC) in our study.

\section{CONCLUSION}

The modification of the flow patterns and the occurrence of the defects induced by bubbles injection in a Taylor-Couette flow with rotation of the inner cylinder has been experimentally studied. It is the first time that the problematic of the dynamics of spatiotemporal defects in a Taylor-Couette flow is addressed for a bubbly mixture. For the Reynolds number range of the study, the basic flow (single-phase flow) is a turbulent Taylor vortex flow, for which the Taylor vortices are arranged as a steady toroidal pattern, axially periodic, without waviness in the azimuthal direction. For the bubble size of the study, bubbles are captured by the Taylor vortices and in the outflow region between the vortices. Thus, the Taylor vortices arrangement has been characterized based on visualizations of the bubbles arrangement. Using the statistical tools applied to the space-time diagrams of the bubbles patterns in the axial direction, we have evidenced that bubbles can modify the arrangement of the Taylor vortices: (a) bubbles entrapment modify the axial wavelength, (b) bubbles entrapment in the vortices can bring about the occurrence of an azimuthal wave, (c) for particular conditions of the Reynolds number and volumetric fraction, under gravity effect, bubbles entrapment can connect the Taylor vortices (spiral pattern), (d) bubbles are responsible for the nucleation of spatiotemporal defects (merging or splitting of the Taylor vortices) which results from a switching between basic patterns such as toroidal and spiral patterns (composite patterns flow). We have shown that the composite patterns flow can be classified as three regimes: (i) the structured 
composite patterns regime SCP characterized by punctual periodic defects, (ii) the intermittency defect chaos regime IDC for which intermittent defects spots alternate with the basic patterns in time and space, and (iii) the developed defects chaos regime DDC characterized by a large defects density and destructured patterns. To our knowledge, it is the first time that unstructured composite bubbly flows IDC and DDC are evidenced. Using the complex demodulation of the space-time diagrams, the defects have been characterized in the transition from SCP to DDC. It is shown that the development of the defects in the transition obeys the DMT theory (defect mediated turbulence theory), as for viscoelastic fluids [29]. It has been evidenced that the available air volumetric fraction $\alpha$ is the adequate control parameter to describe the transition scenario of the DMT in the framework theory of Ginzburg-Landau equation. $\alpha$ results from a combination of the Reynolds number and the air injection rate. This is different from viscoelastic fluids for which the control parameter of the DMT is the Reynolds number [29]. The evolution of the number of defects with $\alpha$ fitted by the equation of Egolf-Greenside has been used to determine the threshold value of $\alpha$ that characterizes the occurrence of the DMT $\left(\alpha_{\mathrm{def}}=0.0016 \%\right)$. The evolution with $\alpha$ of the correlation length and time of the DMT patterns has been used to determine the threshold value of $\alpha$ for the transition between the SCP and IDC regimes $\left(\alpha_{\text {IDC }}=0.005 \%\right)$. This transition value was confirmed by the covariance of the separation time between two consecutives defects. The transition from IDC to the DDC has been evidenced at $\alpha_{\mathrm{DDC}}=0.01 \%$. This transition is characterized by: (i) a correlation length $\xi$ of the patterns of the same order as the mean distance $H_{\text {sep }}$ between defects $\left(\xi=H_{\text {sep }}\right)$, (ii) a mean separation time between two consecutive defects of the same order as the lifetime of defects, (iii) an axial diffusion velocity of the bubbles patterns of the same order as the bubbles rising velocity. In the DDC regime, due to our finite geometry, at high air volumetric fractions $(\alpha>0.02 \%)$, we observe a saturation of the number of defects, as well as a saturation of the diffusion rate, correlation time and length. At saturation, bubbles upward jumping control the dynamics of the defects. The parameters of influence are the bubbles terminal rising velocity and the bubbles equilibrium positions in the vortices, under gravity effect. Generally speaking, we have shown that by introducing defects, bubbles can modify the mixing properties of the Taylor-Couette flow. Nevertheless, for same range of the Reynolds numbers, same range of the air volumetric fraction, same viscosity and same bubble size, a change in the geometry of the device (small or large gap), by changing the circulation of the Taylor vortices, can result in different equilibrium positions of the bubbles capture, which is expected to change the axial wavelength and the dynamics of the defects. Also we have shown that gravity effect induced by the bubbles terminal rising velocity plays a role in the DMT transitions. Thus, we can expect different critical values of the air volumetric fraction that characterize the transitions of the DMT in a Taylor bubbly flow, when changing the viscosity, bubble size or the geometry of the gap. These aspects should be investigated in future studies.

\section{ACKNOWLEDGEMENTS}

This work was carried out at IRENav and subsidized by the French Naval Academy. The authors are grateful to the technical staff of the French Naval Academy.

[1] A. du Cluzeau, G. Bois, and A. Toutant, Analysis and modeling of Reynolds stresses in turbulent bubbly up-flows from direct numerical simulations, J. Fluid Mech. 866, 32 (2019).

[2] S. Hosokawa and A. Tomiyama, A Bubble-induced pseudo turbulence in laminar pipe flows, Inter. J. Heat Fluid Flow 40, 97 (2013).

[3] A. Ferrante and S. Elgobashi, On the physical mechanisms of drag reduction in a spatially developing turbulent boundary layer laden with microbubbles, J. Fluid Mech. 503, 345 (2004).

[4] J. lu, A. Fernandez, and G. Tryggvason, The effect of bubble on the wall drag in a turbulent chanel flow, Phys. Fluids 17, 95102 (2005). 
[5] H. Djeridi, C. Gabillet, and J. Y. Billard, Two-phase Couette-Taylor flow: Arrangement of dispersed phase and effects on the flow structures, Phys. Fluids 16, 128 (2004).

[6] T. Watamura, Y. Tasaka, and Y. Muraï, Intensified and attenuated waves in a microbubble Taylor-Couette flow, Phys. Fluids 25, 054107 (2013).

[7] T. H. Van den Berg, S. Luther, D. P. Lathrop, and D. Lohse, Drag Reduction in Bubbly Taylor-Couette Turbulence, Phys. Rev. Lett. 94, 044501 (2005).

[8] Y. Murai, H. Oiwa, and Y. Takeda, Frictional drag reduction in bubbly Taylor-Couette flow, Phys. Fluids 20, 034101 (2008).

[9] G. N. Fokoua, C. Gabillet, A. Aubert, and C. Colin, Effect of bubbles arrangement on the viscous torque in bubbly Taylor-Couette flow, Phys. Fluids 27, 034105 (2015).

[10] E. Climent, M. Simonnet, and J. Magnaudet, Preferential accumulation of bubbles in Couette-Taylor flow patterns, Phys. Fluids 19, 083301 (2007).

[11] A. Mehel, C. Gabillet, and H. Djeridi, Analysis of the flow pattern modifications in a bubbly CouetteTaylor flow, Phys. Fluids 19, 118101 (2007).

[12] A. Chouippe, E. Climent, D. Legendre, and C. Gabillet, Numerical simulation of bubble dispersion in turbulent Taylor-Couette flow, Phys. Fluids 26, 43304 (2014).

[13] K. Yoshida, Y. Tasaka, Y. Murai, and T. Takeda, Mode transition in bubbly Taylor-Couette flow measured by PTV, J. Phys.: Conf. Ser. 147, 012013 (2009).

[14] Y. Murai, Y. Tasaka, Y. Oishi, and Y. Takeda, Modal switching of bubbly Taylor-Couette flow investigated by tracking velocimetry, Exp. Fluids 59, 164 (2018).

[15] B. van Ruymbeke, Y. Murai, Y. Tasaka, Y. Oishi, C. Gabillet, C. Colin, and N. Latrache, Quantitative visualization of swirl and cloud bubbles in Taylor-Couette flow, J. Visual. 20, 349 (2017).

[16] S. T. Wereley and R. M. Lueptow, Velocity field for Taylor-Couette flow with an axial flow, Phys. Fluids 11, 12 (1999).

[17] K. Atken, J. Fontain, and J. E. Wesfreid, Highly turbulent Couette-Taylor bubbly flow patterns, J. Fluid Mech. 422, 55 (2000).

[18] P. Coullet, L. Gil, and J. Lega, Defect-Mediated Turbulence, Phys. Rev. Lett. 62, 1619 (1989).

[19] M. C. Cross and P. C. Hohenberg, Pattern formation outside equilibrium, Rev. Mod. Phys. 65, 851 (1993).

[20] P. Cerisier, R. Occelli, C. Perez-Garcia, and C. Jamond, Structural disorder in Bénard-Marangoni convection, J. Physique 48, 569 (1987).

[21] A. Kudrolli and J. P. Gollub, Localized spatiotemporal chaos in surface waves, Phys. Rev. E 54, R1052 (1996).

[22] I. Shani, G. Cohen, and J. Fineberg, Localized Instability on the Route to Disorder in Faraday Waves, Phys. Rev. Lett. 104, 184507 (2010).

[23] P. Bot and I. Mutabazi, Dynamics of spatiotemporal defects in the Taylor-Dean system, Eur. Phys. J. B 13, 141 (2000).

[24] K. E. Daniels and E. Bodenschatz, Defect Turbulence in Inclined Layer Convection, Phys. Rev. Lett. 88, 034501 (2002).

[25] A. Cros and P. Le Gal, Defect turbulence in a spiral wave pattern in the torsional Couette flow, Phys. Rev. E 70, 016309 (2004).

[26] A. B. Ezersky, N. Abcha, and I. Mutabazi, The structure of spatiotemporal defects in a spiral pattern in the Couette-Taylor flow, Phys. Lett. A 374, 3297 (2010).

[27] I. Rehberg, S. Rasenat, and V. Steinberg, Traveling Waves and Defect-Initiated Turbulence in Electroconvecting Nematics, Phys. Rev. Lett. 62, 756 (1989).

[28] G. Goren, I. Porcaccia, S. Rasenat, and V. Steinberg, Interactions and Dynamics of Topological Defects: Theory and Experiments Near the Onset of Weak Turbulence, Phys. Rev. Lett. 63, 1237 (1989).

[29] N. Latrache, N. Abcha, O. Crumeyrolle, and I. Mutabazi, Defect-mediated turbulence in ribbons of viscoelastic Taylor-Couette flow, Phys. Rev. E 93, 043126 (2016).

[30] B. Shraiman, A. Pumir, W. Van Saarloos, P. Hohenberg, H. Chaté, and M. Holen, Spatiotemporal chaos in the one-dimensional complex Ginzburg-Landau equation, Physica D 57, 241 (1992).

[31] D. A. Egolf and H. S. Greenside, Characterization of the Transition from Defect- to Phase-Turbulence, Phys. Rev. Lett. 74, 1751 (1995). 
[32] T. Maxworthy, C. Gnamn, M. Kürten, and F. Durst, Experiments on the rise of air bubbles in clean viscous liquids, J. Fluid Mech. 321, 421 (1996).

[33] A. Mehel, Etude expérimentale d'un écoulement diphasique de Taylor-Couette, Institut de Recherche de l'École Navale, Ph.D. thesis, Université de Nantes, 2006.

[34] G. N. Fokoua, Etude expérimentale de la réduction de traînée par injection de bulles, Institut de Recherche de l'École Navale, Ph.D. thesis, Université Bretagne Occidentale (2013).

[35] D. Coles, Transition in circular Couette flow, J. Fluid Mech. 21, 385 (1965).

[36] P. Castle and F. S. Mobbs, Hydrodynamic stability of the flow between eccentric rotating cylinders: Visual observations and torque measurements, Proc. Inst. Mech. Engrs 182, 41 (1967).

[37] N. Latrache, O. Crumeyrolle, and I. Mutabazi, Transition to turbulence in a flow of a shear-thinning viscoelastic solution in a Taylor-Couette cell, Phys. Rev. E 86, 056305 (2012).

[38] R. Kowe, J. C. Hunt, A. Hunt, B. Couet, and L. J. Bradbury, The effects of bubbles on the volume fluxes and the pressure in unsteady and nonuniform flow of liquids, Int. J. Multiphase Flow 14, 587 (1988).

[39] O. Crumeyrolle, N. Latrache, I. Mutabazi, and A. B. Ezersky, Instabilities with shear-thinning polymer solutions in the Couette-Taylor system, J. Phys.: Conf. Ser. 14, 011 (2005).

[40] V. S. Afraimovich and L. A. Bunimovich, Density of defects and spatial entropy in extended systems, Physica D 80, 277 (1995). 